\title{
COLORING CURVES ON SURFACES
}

\author{
JONAH GASTER ${ }^{1}$, JOSHUA EVAN GREENE ${ }^{2}$ and NICHOLAS G. VLAMIS ${ }^{3}$ \\ ${ }^{1}$ Department of Mathematics and Statistics, McGill University, Montreal, \\ Quebec H3A 0B9, Canada; \\ email: jbgaster@gmail.com \\ ${ }^{2}$ Department of Mathematics, Boston College, Chestnut Hill, MA 02467, USA; \\ email: joshua.greene@bc.edu \\ ${ }^{3}$ Department of Mathematics, Queens College of CUNY, Flushing, NY 11367, USA; \\ email: nicholas.vlamis@qc.cuny.edu
}

Received 30 December 2016; accepted 8 June 2018

\begin{abstract}
We study the chromatic number of the curve graph of a surface. We show that the chromatic number grows like $k \log k$ for the graph of separating curves on a surface of Euler characteristic $-k$. We also show that the graph of curves that represent a fixed nonzero homology class is uniquely $t$-colorable, where $t$ denotes its clique number. Together, these results lead to the best known bounds on the chromatic number of the curve graph. We also study variations for arc graphs and obtain exact results for surfaces of low complexity. Our investigation leads to connections with Kneser graphs, the Johnson homomorphism, and hyperbolic geometry.
\end{abstract}

2010 Mathematics Subject Classification: 57M15 (primary); 05C15 (secondary)

\section{Introduction}

Curve graphs play a central role in the study of mapping class groups, Teichmüller spaces, and 3-manifolds. In this setting, their large-scale geometry has grown into a subject of intensive study [MM99, Bow08, Min10, BCM12, BBF15]. Alongside it, interest has grown in their graph-theoretic properties [Iva97, AL13, MRT14, BM15, KK13]. Here we explore their chromatic number, one of the most natural and attractive invariants in graph theory.

We briefly fix some terminology. Let $S$ denote a compact, connected, orientable surface. The curve graph $\mathcal{C}(S)$ is the graph whose vertices are isotopy classes of essential simple closed curves on $S$, where two isotopy classes are adjacent if they have disjoint representatives [Har81]. (A small adjustment is made when $S$

(c) The Author(s) 2018. This is an Open Access article, distributed under the terms of the Creative Commons Attribution licence (http://creativecommons.org/licenses/by/4.0/), which permits unrestricted re-use, distribution, and reproduction in any medium, provided the original work is properly cited. 
is one of a few low complexity examples.) We refer to the vertices of $\mathcal{C}(S)$ simply as curves. The chromatic number $\chi(G)$ of a graph $G$ is the fewest number of colors required to color the vertices of $G$ so that adjacent vertices get different colors. Thus, our motivating problem is to estimate $\chi(\mathcal{C}(S))$, the fewest number of parts required to partition the curves on $S$ so that any two curves in a given part intersect.

Bestvina, Bromberg, and Fujiwara were the first to study the quantity $\chi(\mathcal{C}(S))$. They showed that it is finite en route to proving that the mapping class group $\operatorname{Mod}(S)$ has finite asymptotic dimension [BBF15, Lemma 5.6]. Curve graphs are locally infinite, so the finite colorability is not at all apparent $a$ priori. Their bound on $\chi\left(\mathcal{C}\left(S_{g}\right)\right)$ for a closed surface of genus $g$ is doubly exponential in $g$, which they did not attempt to optimize. By contrast, a simple lower bound on $\chi(G)$ comes from its clique number $\omega(G)$, the size of the largest complete subgraph. Maximum cliques in $\mathcal{C}(S)$ correspond to pants decompositions of $S$, so $\omega\left(\mathcal{C}\left(S_{g}\right)\right)=3 g-3$ for $g \geqslant 2$. Our work was motivated in part to close the gap between the linear and doubly exponential bounds on $\chi\left(\mathcal{C}\left(S_{g}\right)\right)$.

Another source of motivation comes from the study of topological designs [JMM96]. An attractive unsolved problem in this area is to determine the size of a largest 1-system on $S_{g}$, that is a collection of simple closed curves that pairwise intersect at most once. Denote this value by $N(g)$. Malestein, Rivin, and Theran proved that $g^{2} \lesssim N(g) \lesssim g \cdot 4^{g}$ and that the size of a largest collection of curves that pairwise intersect exactly once is $2 g+1$ [MRT14, Theorems $1.1 \&$ 1.4]. Przytycki dramatically improved the upper bound to $N(g) \lesssim g^{3}$ [Prz15, Theorems $1.2 \& 1.4]$, and Aougab et al. subsequently improved it to $N(g) \lesssim$ $g^{3} /(\log g)^{2}$ [ABG17, Theorem 1.1]. A color class in a proper coloring of the subgraph of $\mathcal{C}\left(S_{g}\right)$ induced on a 1-system is precisely a collection of curves that pairwise intersect exactly once. It follows that $N(g) \leqslant(2 g+1) \chi\left(\mathcal{C}\left(S_{g}\right)\right)$. Therefore, an upper bound $\chi\left(\mathcal{C}\left(S_{g}\right)\right) \ll g^{2} /(\log g)^{2}$ would improve on the best current upper bound on $N(g)$; conversely, large constructions of 1-systems would lead to improved lower bounds on $\chi\left(\mathcal{C}\left(S_{g}\right)\right)$.

1.1. Prelude. We set the stage for our results with a brief, informal account of the case of the $n$-holed sphere $\Sigma_{n}$. See Section 2 for precise definitions of the graphs $\mathrm{KG}(n)$ and $\mathrm{CG}(n)$ appearing here. A curve on $\Sigma_{n}$ partitions its holes into two nonempty parts. Disjoint curves give rise to nested partitions: one part for one curve is properly contained in one part for the other curve. Recording the partitions of the holes and the nesting relation gives a graph homomorphism from $\mathcal{C}\left(\Sigma_{n}\right)$ to a particular finite graph $\mathrm{KG}(n)$. Recall that a graph homomorphism $G \rightarrow H$ is a map from the vertex set of $G$ to the vertex set of $H$ that preserves adjacency. For instance, the special case in which $H$ is a complete graph $K_{t}$ 
corresponds to a $t$-coloring of $G$. On the other hand, arranging the holes of $\Sigma_{n}$ around a circle, we obtain a finite subgraph $\mathrm{CG}(n)$ inside $\mathcal{C}\left(\Sigma_{n}\right)$ of those curves that surround a cyclic interval of holes. (For convenience, in Section 1.1, we let $\mathrm{CG}(n)$ denote the graph $\mathrm{CG}(n) \backslash \mathrm{CG}(n, 1)$ defined in Section 2.) We thereby obtain a sequence of homomorphisms $\mathrm{CG}(n) \rightarrow \mathcal{C}\left(\Sigma_{n}\right) \rightarrow \operatorname{KG}(n)$, the first of which is an embedding. Since homomorphisms compose, it follows that $\chi(\mathrm{CG}(n)) \leqslant \chi\left(\mathcal{C}\left(\Sigma_{n}\right)\right) \leqslant \chi(\mathrm{KG}(n))$. The graphs $\mathrm{KG}(n)$ and $\mathrm{CG}(n)$ are natural unions of the well-studied two-parameter families of $\operatorname{Kneser}$ graphs $\operatorname{KG}(n, k)$ and cyclic interval graphs $\mathrm{CG}(n, k)$. As we show, their chromatic numbers both grow like $n \log n$, and in fact are within a factor of $\ln (2) \approx 0.69$ apart. In this way, we obtain a very precise estimate on $\chi\left(\mathcal{C}\left(\Sigma_{n}\right)\right)$.

The graph $\mathrm{CG}(n)$ repeatedly arises as a subgraph of arc and curve graphs, as does $\mathrm{KG}(n)$ as a target for homomorphisms from these graphs. The tight control we gain over the (fractional) chromatic numbers of $\mathrm{CG}(n)$ and $\mathrm{KG}(n)$ underpins many of our results for curve graphs. Other researchers have used the graph $\mathrm{CG}(n)$ as a probe of the large-scale geometry of the curve complex $\mathcal{C}\left(\Sigma_{n}\right)$. Embeddings of $\operatorname{CG}(n)$ into $\mathcal{C}\left(\Sigma_{n}\right)$ are rigid in the sense that any two are related by an automorphism of $\mathcal{C}\left(\Sigma_{n}\right)$ [AL13]. The induced subcomplex on $\mathrm{CG}(n)$ is homeomorphic to an $(n-4)$-dimensional sphere [Lee89], and it represents a generator of the homology of $\mathcal{C}\left(\Sigma_{n}\right)$ as a $\operatorname{Mod}^{ \pm}\left(\Sigma_{n}\right)$-module [BBM15]. Thus, it is remarkable-or not? - that it also accounts for the order of growth of $\chi\left(\mathcal{C}\left(\Sigma_{n}\right)\right)$. We note that the maximal clique graph $\mathcal{K}(\mathrm{CG}(n))$ (see Section 1.3) is isomorphic to the associahedron or the flip graph on the triangulations of an $n$-gon. The determination of the chromatic number of $\chi(\mathcal{K}(\mathrm{CG}(n)))$ is a fascinating open problem: it is not even known whether it grows unbounded in $n\left[\mathbf{F M F P H}{ }^{+} \mathbf{0 9}\right.$, Sections 4 \& 6], [STT88].

1.2. Statement of results. In Section 2, we introduce the total Kneser graph $\mathrm{KG}(n)$ and the total cyclic interval graph $\mathrm{CG}(n)$. In spite of how naturally they arise in the present setting, it appears that $\operatorname{KG}(n)$ has not been studied before, nor has $\mathrm{CG}(n)$ from the graph-theoretic perspective. We determine the order of growth of their (fractional) chromatic numbers:

THEOREM 1.1. The fractional and ordinary chromatic numbers of $\mathrm{CG}(n)$ and $\mathrm{KG}(n)$ are all $\sim n \log n$.

(See Section 1.3 for the notation $\lesssim$ and $\sim$ and Section 2 for the definition of fractional chromatic number.) Moreover, the implied constants in the bounds on $\chi(\mathrm{KG}(n))$ are within a factor of $\ln (2)$. The determination of the chromatic number of the ordinary Kneser graph $\operatorname{KG}(n, k)$ was the content of a famous conjecture 
due to Kneser [Kne55] and settled in a celebrated theorem of Lovász [Lov78]. Kneser exhibited a proper coloring of $\operatorname{KG}(n, k)$ using $n-2 k+2$ colors, and Lovász proved its optimality by defining the neighborhood complex $N(G)$ of a graph $G$, showing that the connectivity of $N(G)$ bounds $\chi(G)-3$ from below, and applying this bound to $\mathrm{KG}(n, k)$. By contrast, the chief difficulty in Theorem 1.1 lies in establishing the upper bound on $\chi(\operatorname{KG}(n))$, which we accomplish by a variation on Kneser's original coloring of $\operatorname{KG}(n, k)$.

In Section 3, we formalize the argument from the Prelude and apply Theorem 1.1 in order to determine the order of growth of the chromatic number of the curve graph of the $n$-holed sphere $\Sigma_{n}$ :

THEOREM 1.2. The fractional and ordinary chromatic numbers of $\mathcal{C}\left(\Sigma_{n}\right)$ are $\sim n \log n$.

Prior to our work, Radhika Gupta obtained the estimate $\chi\left(\mathcal{C}\left(\Sigma_{n}\right)\right) \lesssim n^{2}$ (R. Gupta, private communication).

In Section 4, we generalize Theorem 1.2 to an estimate on the chromatic number of $\mathcal{C}_{\text {sep }}(S)$, the subgraph of $\mathcal{C}(S)$ induced on the separating curves:

THEOREM 1.3. If $S$ has Euler characteristic $-k<0$, then $\chi\left(\mathcal{C}_{\text {sep }}(S)\right) \sim k \log k$.

As with the case of a planar surface, the bounds come from embedding a cyclic interval graph into $\mathcal{C}_{\text {sep }}(S)$ and mapping it to a Kneser graph. However, the homomorphism to the Kneser graph is subtler in this more general setting. Drawing inspiration from [BP07], we place a hyperbolic metric on $S$ compatible with a pants decomposition in which all of the pant cuffs are very short. The simple closed geodesics on this hyperbolic surface congregate near a 1-complex whose complement consists of $12 k$ regions. A separating simple closed geodesic partitions these regions into two parts, and disjoint geodesics yield distinct nested partitions. In this way, we obtain the required homomorphism to the Kneser graph $\mathrm{KG}(12 k)$. Theorem 4.1 contains the precise statement that we require, and we give a careful argument through a sequence of lemmas in hyperbolic geometry. Ian Biringer has suggested an alternate description of this homomorphism based instead on train tracks, thereby eliminating the need for hyperbolic geometry in proving Theorem 1.3.

In Section 5, we study the subgraph $\mathcal{C}_{v}(S)$ of $\mathcal{C}(S)$ induced on the curves that represent a fixed nonzero, primitive homology class $v \in H_{1}(S ; \mathbb{Z})$. In this case, we obtain not only an exact answer but also a uniqueness result:

THEOREM 1.4. For any $v \neq 0, \mathcal{C}_{v}(S)$ is uniquely $t$-colorable, where $t$ denotes the clique number of $\mathcal{C}_{v}(S)$. 
See Theorems 5.1, 5.12, and 5.16 for more precise statements. The proof of Theorem 1.4 relies on a different set of techniques than those appearing up to this point. The color of a curve in $\mathcal{C}_{v}(S)$ is based on the genus $h$ of an immersed subsurface that it cobounds with a fixed reference curve in its homology class: for instance, when $S$ is closed and has genus $g$, the color is the value $h(\bmod g-1)$. In fact, the coloring coincides with the signed length introduced by Irmer [Irm15, Section 4.1]. As a byproduct, the coloring permits an interpretation of signed length in terms of genera of immersed surfaces in the case of a closed surface; moreover, the use of domains in Section 5.2 makes it easy to calculate. To prove that the coloring of $\mathcal{C}_{v}(S)$ is unique, we show that it is possible to connect any two maximal cliques through a sequence in which each consecutive pair meet in all but one vertex: in other words, the maximal clique graph $\mathcal{K}\left(\mathcal{C}_{v}(S)\right)$ is connected. We do so by studying the action of a relevant mapping class group on $\mathcal{K}\left(\mathcal{C}_{v}(S)\right)$ and applying Putman's trick [Put08].

On combination of Theorems 1.3 and 1.4, we bound the chromatic number of the curve graph of a closed surface as follows:

THEOREM 1.5. $g \cdot \log g \lesssim \chi\left(\mathcal{C}\left(S_{g}\right)\right) \lesssim g \cdot 4^{g}$.

The lower bound comes simply from separating curves. The upper bound comes from partitioning curves according to their (mod 2) homology classes and coloring the curves in each class separately. The zero class consists of the separating curves, and each of the $4^{g}-1$ nonzero classes $\bar{v}$ are disjoint unions of the graphs $\mathcal{C}_{v}\left(S_{g}\right)$, where $v$ reduces to $\bar{v}(\bmod 2)$. Theorem 1.4 and a small maneuver around the axiom of countable choice (Proposition 5.10) lets us color all the curves representing $\bar{v}$ by $g-1$ colors. The upper bound in Theorem 1.5 then follows.

Thus, the chromatic number of $\mathcal{C}\left(S_{g}\right)$ is superlinear and at most exponential in $g$. These bounds leave great room for improvement, and we suspect the truth lies closer to the lower bound. On the other hand, a construction of 1 -systems on $S_{g}$ of size larger than $g^{2} \cdot \log g$ would lead to an improvement on our lower bound.

In Section 6, we explore a consequence of Theorem 1.4 for a closed surface $S=S_{g}$. Set $H=H_{1}(S, \mathbb{Z})$ and let $\mathcal{I}<\operatorname{Mod}(S)$ denote the Torelli group, the mapping classes that act trivially on $H$. For each primitive nonzero class $v \in H$, the group $\mathcal{I}$ acts by automorphisms on $\mathcal{C}_{v}(S)$. By uniqueness of the minimal coloring, it permutes the color classes in a minimal coloring. In fact, it permutes them cyclically, and the action determines a homomorphism

$$
\chi: \mathcal{I} \rightarrow \operatorname{Hom}(H, \mathbb{Z} /(g-1) \mathbb{Z})
$$


(Lemma 6.2). We explicitly compute the action of generators for $\mathcal{I}$ on the color classes, and we obtain a relationship between the coloring of $\mathcal{C}_{v}(S)$ and the Johnson homomorphism. The latter is a homomorphism

$$
\tau: \mathcal{I} \rightarrow \operatorname{Hom}\left(H, \bigwedge^{2} H\right) / H
$$

that captures the free part of $H_{1}(\mathcal{I} ; \mathbb{Z})$. Johnson showed that the composition of $\tau$ with the algebraic intersection pairing $\bigwedge^{2} H \rightarrow \mathbb{Z}$ and reduction $(\bmod g-1)$ gives the Chillingworth homomorphism $t: \mathcal{I} \rightarrow \operatorname{Hom}(H, \mathbb{Z} /(g-1) \mathbb{Z})[$ Chi72a, Chi72b, Joh80]. We show:

THEOREM 1.6. The color permutation homomorphism $\chi$ equals the Chillingworth homomorphism $t$.

In fact, the identification of the coloring of $\mathcal{C}_{v}(S)$ with Irmer's signed length immediately implies that the coloring homomorphism $\chi$ is equal to her stable length homomorphism $\phi$ [Irm15, Lemma 5]. Irmer proves Theorem 1.6 with $\phi$ in place of $\chi$ [Irm15, Theorem 1], and our proof mimics hers. Thus, the novelty in Theorem 1.6 compared to Irmer's work is its interpretation in terms of coloring. As a result, in Corollary 6.4 we recast the coloring of $\mathcal{C}_{v}(S)$ in terms of $t$.

In Section 7, we explore analogues of the preceding results for arc graphs. The vertices of the arc graph $\mathcal{A}(S)$ are isotopy classes of essential properly embedded $\operatorname{arcs}$ on $S$, and two classes are adjacent if they have disjoint representatives. Here the isotopy is free on the boundary. We specialize to the case of a planar surface. We show that the chromatic number of the subgraph $\mathcal{A}_{\text {sep }}\left(\Sigma_{n}\right)$ induced on the separating arcs grows like $n \log n$ (Theorem 7.3) and the subgraph of $\mathcal{A}\left(\Sigma_{n}\right)$ induced on arcs representing a fixed nonzero homology class is uniquely $(n-2)$ colorable (Theorem 7.4). The analogue to Theorem 1.5 in this setting is:

THEOREM 1.7. $n \log n \lesssim \chi\left(\mathcal{A}\left(\Sigma_{n}\right)\right) \lesssim n^{3}$

The fact that the upper bound is polynomial and not exponential derives from the fact that the number of (mod 2) homology classes of arcs on $\Sigma_{n}$ is quadratic in $n$, whereas the number of $(\bmod 2)$ homology classes of curves on $S_{g}$ is exponential in $g$. Doubling $\Sigma_{n}$ along its boundary induces an inclusion $\mathcal{A}\left(\Sigma_{n}\right) \hookrightarrow \mathcal{C}\left(S_{n-1}\right)$. Thus, an improved lower bound on $\chi\left(\mathcal{A}\left(\Sigma_{n}\right)\right)$ would result in a corresponding improvement on $\chi\left(\mathcal{C}\left(S_{g}\right)\right)$, and conversely for the upper bounds. It seems likely, albeit less direct, that an improvement on the lower bound on $\chi\left(\mathcal{C}\left(S_{g}\right)\right)$ would inform one on $\chi\left(\mathcal{A}\left(\Sigma_{n}\right)\right)$, and conversely for the upper bounds.

In Sections 8 and 9, we obtain exact results for surfaces of low complexity. In Section 8 , we study $\mathcal{A}\left(\Sigma_{4}\right)$ and various subgraphs of it. For example, we 
show that the subgraph of $\mathcal{A}\left(\Sigma_{4}\right)$ induced on arcs with exactly one endpoint on a fixed boundary component has chromatic number 4 , with color classes corresponding to the orbits under the action of the level-3 congruence subgroup $\Gamma(3)<\operatorname{PSL}(2, \mathbb{Z})$ on $P^{1}\left(\mathbb{Z}^{2}\right)$ (Theorem 8.6). In Section 9, we study $\mathcal{C}\left(S_{2}\right)$ and its subgraph $\mathcal{N}\left(S_{2}\right)$ induced on the nonseparating curves. Using the hyperelliptic involution, we show:

THEOREM 1.8. $\chi\left(\mathcal{N}\left(S_{2}\right)\right)=4$ and $\chi\left(\mathcal{C}\left(S_{2}\right)\right)=5$.

Finally, in Section 10, we collect some questions for further study.

1.3. Conventions and notation. If $f$ and $g$ denote two real-valued functions, then we write $f \lesssim g$ if there exists an absolute constant $C>0$ such that $f \leqslant C \cdot g$. We write $f \sim g$ if $f \lesssim g$ and $g \lesssim f$.

All surfaces appearing in our results are compact, connected, and orientable. We denote by $S_{g}^{b}$ a surface of genus $g$ with $b$ boundary components, or holes. If $b=0$, then we suppress it from the notation, and if $g=0$, then we write $\Sigma_{n}=S_{0}^{n}$.

The mapping class group of $S$ is the $\operatorname{group} \operatorname{Mod}(S):=\pi_{0}\left(\mathrm{Homeo}^{+}(S)\right)$. For convenience, we break slightly with the convention of [FM11] by allowing $S$ to permute boundary components; the subgroup acting trivially on boundary components is denoted by $\operatorname{PMod}(S)$. This difference is relevant in the proofs of Proposition 5.13 and Theorem 7.4.

A simple closed curve on a surface is separating if its complement is disconnected; it is peripheral if it is isotopic to a hole; and it is essential if it neither is peripheral nor bounds a disk. Similarly, a homology class is separating or peripheral if it is represented by an oriented simple closed curve with the corresponding property.

We denote the curve (respectively arc) graph by $\mathcal{C}(S)$ (respectively $\mathcal{A}(S)$ ), and the subgraphs induced by separating and nonseparating curves (respectively arcs) by $\mathcal{C}_{\text {sep }}(S)$ and $\mathcal{N}(S)$ (respectively $\mathcal{A}_{\text {sep }}(S)$ and $\mathcal{A} \mathcal{N}(S)$ ). Given a homology class $v$ (respectively relative to $\partial S$ ), we denote the subgraph spanned by curves (respectively arcs) which can be oriented to be homologous to $v$ by $\mathcal{C}_{v}(S)$ (respectively $\mathcal{A}_{v}(S)$ ).

When convenient, we elide the difference between a graph and the flag simplicial complex with the same 1-skeleton (in which any complete subgraph on $k$ vertices spans a unique $(k-1)$-simplex). For example, $\mathcal{C}(S)$ may also denote the curve complex associated to $S$.

Given a simplicial complex $\mathcal{C}$, we let $\mathcal{K}(\mathcal{C})$ denote the graph whose vertices consist of the maximal simplices in $\mathcal{C}$, where two maximal simplices are adjacent if they meet in a codimension- 1 face. We refer to $\mathcal{K}(\mathcal{C})$ as the maximal clique 
graph of $\mathcal{C}$. The reader is cautioned that $\mathcal{K}\left(\mathcal{C}\left(S_{g}\right)\right)$ is not quite the 'clique graph' in the sense of $[K K 13$, p. 3], in which vertices correspond to (not necessarily maximal) cliques.

\section{Kneser graphs and cyclic interval graphs}

In this section, we introduce the total Kneser graph $\mathrm{KG}(n)$ and its subgraph the total cyclic interval graph $\mathrm{CG}(n)$. These graphs are natural unions of the well-known $\mathrm{Kneser}$ graphs $\mathrm{KG}(n, k)$ and cyclic interval graphs $\mathrm{CG}(n, k)$. We determine the fractional chromatic numbers of these graphs and the growth orders of their chromatic numbers in Theorems 2.3 and 2.5. For more background on graph theory, including discussion about homomorphisms, (fractional) chromatic numbers, and the two-parameter Kneser and cyclic interval graphs, see [GR13, Ch. 7].

We recall and introduce some important notation. Let $G$ denote a graph. A clique in $G$ is a collection of pairwise adjacent vertices, that is the vertices of a complete subgraph, and an independent set in $G$ is a collection of pairwise nonadjacent vertices. The clique number $\omega(G)$ is the maximum size of a clique in $G$. A proper coloring of $G$ is a partition of its vertex set into independent sets, which are called the color classes of the coloring. The chromatic number $\chi(G)$ is the fewest number of color classes in a proper coloring of $G$.

Linear programming offers the following perspective. A clique can be interpreted as a $0 / 1$ weighting of the vertices of $G$ with the property that the sum of the weights of the vertices in any independent set is at most one. The clique number is thus the maximum, over all cliques of $G$, of the sum of the weights of the vertices. Similarly, a coloring can be interpreted as a $0 / 1$ weighting of the independent sets of $G$ with the property that the sum of the weights on the independent sets containing any fixed vertex is one. The chromatic number is thus the minimum, over all colorings of $G$, of the sum of the weights of the independent sets.

These are integer linear programs, and they admit the following real-valued relaxations. A fractional clique of $G$ is a weighting of its vertices by nonnegative real numbers subject to the condition that the sum of the weights on any independent set is at most one. The fractional clique number $\omega_{f}(G)$ is the supremum, over all fractional cliques of $G$, of the sum of the weights on the vertices. A fractional coloring of $G$ is a weighting of its independent sets by nonnegative real numbers subject to the condition that the sum of the weights on the independent sets containing any fixed vertex $v$ is at least one. The fractional chromatic number $\chi_{f}(G)$ is the infimum, over all fractional colorings of $G$, of the sum of the weights on the independent sets. There are straightforward bounds 
$\omega(G) \leqslant \omega_{f}(G) \leqslant \chi_{f}(G) \leqslant \chi(G)$, and linear programming duality has the corollary that $\omega_{f}(G)=\chi_{f}(G)$.

Given a pair of positive integers $n \geqslant 2 k$, the Kneser graph $\operatorname{KG}(n, k)$ is the graph whose vertices are the $k$-element subsets of $\{1, \ldots, n\}$ and whose edges are unordered pairs of disjoint subsets. A cyclic interval is a cyclic shift of the set $\{1, \ldots, k\}$ modulo $n$. The cyclic interval graph $\mathrm{CG}(n, k)$ is the subgraph of $\mathrm{KG}(n, k)$ induced on the cyclic intervals.

The fractional chromatic numbers of the Kneser graphs and cyclic interval graphs are well known [GR13, Section 7.7], and the determination of the chromatic number of the Kneser graph $\operatorname{KG}(n, k)$ is a celebrated theorem of Lovász [Lov78]. We record these values here:

ThEOREM 2.1. $\chi_{f}(\mathrm{CG}(n, k))=\chi_{f}(\operatorname{KG}(n, k))=n / k, \chi(\mathrm{CG}(n, k))=\lceil n / k\rceil$, and $\chi(\operatorname{KG}(n, k))=n-2 k+2$.

Given a positive integer $n \geqslant 2$, the total Kneser graph $\operatorname{KG}(n)$ is the graph whose vertices are partitions of $\{1, \ldots, n\}$ into an unordered pair of nonempty disjoint subsets $(A, B)$. We often express a partition just by one of its parts, since there is no loss of information. Two such partitions $(A, B),(C, D)$ are nested if one of $A$ or $B$ is contained in one of $C$ or $D$; note that the condition is symmetric in the two pairs. The edges of $\mathrm{KG}(n)$ are pairs of distinct nested partitions. The total cyclic interval graph $\mathrm{CG}(n)$ is the subgraph of $\mathrm{KG}(n)$ induced on partitions in which the parts are cyclic intervals. Observe that for $k<n / 2, \operatorname{KG}(n, k) \subset$ $\mathrm{KG}(n)$ is induced on the partitions $(A, B)$ with $\min \{|A|,|B|\}=k$, and $\mathrm{CG}(n, k)=\mathrm{CG}(n) \cap \mathrm{KG}(n, k)$.

A cyclic interval contains a minimal element, the shift of 1 . We label a vertex $(A, B) \in \mathrm{CG}(n)$ by the pair $(i, j)$ that records the minimal elements of $A$ and $B$, with the convention that $i<j$. Two such pairs $(i, j)$ and $\left(i^{\prime}, j^{\prime}\right)$ are linked if $i<i^{\prime}<j<j^{\prime}$ or $i^{\prime}<i<j^{\prime}<j$. Under the labeling by pairs, edges in CG(n) correspond precisely to unlinked pairs.

LEMmA 2.2. If $S$ is an independent set in $\operatorname{CG}(n)$, then $|S| \leqslant \min \{|A| \mid(A$, $B) \in S\}$.

Proof. Choose any $(A, B) \in S$. By applying a cyclic shift to $\mathrm{CG}(n)$, we may assume that $j-i=|A|$, where $(A, B)$ gets labeled $(i, j)$. Since $S$ is an independent set, the labels in $S$ are pairwise linked. Select any other label $\left(i^{\prime}, j^{\prime}\right)$ in $S$. Note that exactly one of $i^{\prime}$ and $j^{\prime}$ lies between $i$ and $j$. Furthermore, no two labels in $S$ share a common coordinate. It follows that there are at most $j-i-1$ labels in $S$ that link with $(i, j)$, so $|S| \leqslant j-i=|A|$, as desired. 
For a positive integer $m$, let $H_{m}$ denote the $m$ th harmonic number $\sum_{k=1}^{m}(1 / k)$, and let $p(m) \in\{0,1\}, p(m) \equiv m(\bmod 2)$. Recall the bounds $\log (m)<H_{m} \leqslant$ $\log (m)+1$ for the following result.

Theorem 2.3. $\chi_{f}(\mathrm{KG}(n))=\chi_{f}(\mathrm{CG}(n))=n \cdot H_{\lfloor(n-1) / 2\rfloor}+(1-p(n)) \sim n \log (n)$.

Proof. The fractional chromatic number of $\operatorname{KG}(n)$ is bounded above by the sum of the fractional chromatic numbers of its vertex-disjoint induced subgraphs $\mathrm{KG}(n, k), 1 \leqslant k<n / 2$, and, if $n$ is even, the independent set induced on the partitions into $n / 2$-subsets. Since $\chi_{f}(\operatorname{KG}(n, k))=n / k$ by Theorem 2.1, we obtain the required upper bound.

Next, define $w: V(\mathrm{CG}(n)) \rightarrow \mathbb{R}_{\geqslant 0}$ by $w((A, B))=1 /|A|$, where $|A| \leqslant|B|$. Let $S$ be an independent set in $\operatorname{CG}(n)$. Select $(A, B) \in S$ with $|A|$ minimal. The sum of the weights on the vertices of $S$ is $w(S) \leqslant|S| \cdot w(A) \leqslant|A| /|A|=1$, using Lemma 2.2 in the second inequality. Therefore, $w$ is a fractional clique. Its total value equals the required lower bound.

REMARK 2.4. The fractional clique defined in the proof of Theorem 2.3 is simply the sum of the optimal fractional cliques for the subgraphs $\operatorname{CG}(n, k)$ that get used to establish their fractional chromatic numbers (Theorem 2.1).

The union bound leads to the soft estimate

$$
\chi(\operatorname{KG}(n)) \leqslant \sum_{k=1}^{\lfloor n / 2\rfloor} \chi(\operatorname{KG}(n, k))=\sum_{k=1}^{\lfloor n / 2\rfloor}(n-2 k+2) \sim n^{2},
$$

which grows faster than $\chi_{f}(\operatorname{KG}(n))$. The following theorem uses a refined coloring to show that $\chi_{f}(\mathrm{KG}(n))$ and $\chi(\mathrm{KG}(n))$ grow at the same rate, and in fact differ by a factor of no more than $\ln (2) \approx 0.69$. It is a variation on Kneser's original $(n-2 k+2)$-coloring of $\mathrm{KG}(n, k)$.

THEOREM 2.5. $\chi(\mathrm{KG}(n)) \leqslant n \cdot\left\lceil\log _{2}(n / 2)\right\rceil+1 \sim n \log (n)$.

Proof. Select a subset $A \subset\{1, \ldots, n\}$ with $|A| \leqslant n / 2$. We can uniquely express $|A|=2^{k+1}-l$, where $k, l \in \mathbb{Z}, k \geqslant 0$, and $1 \leqslant l \leqslant 2^{k}$. Let $a$ denote the $l$ th largest element of $A$. Assign $A$ the color consisting of the pair $(k, a)$. See Figure 1 . Observe that this coloring uses $n \cdot\left\lceil\log _{2}(n / 2)\right\rceil$ colors, unless $n$ is a power of 2 . If it is, then we alter the coloring on the $n / 2$-element subsets by giving them all the same color, distinct from those used on the subsets with fewer elements. In this case, the coloring uses $n \cdot\left\lceil\log _{2}(n / 2)\right\rceil+1$ colors. We claim in either case that 


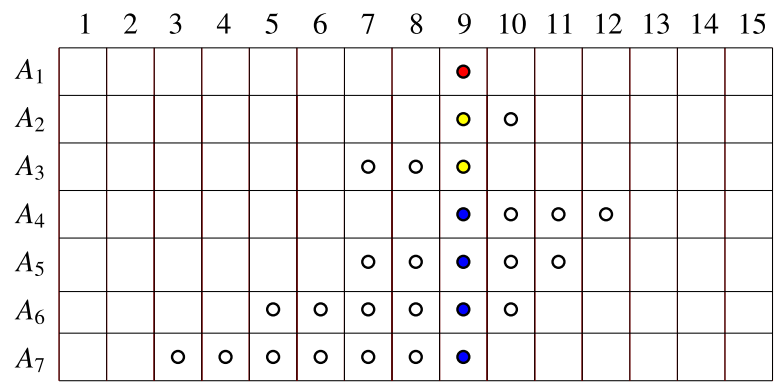

Figure 1. Vertices $A_{i} \in \mathrm{KG}(15),\left|A_{i}\right|=i, i=1, \ldots, 7$. $A_{1}$ gets color $(0,9) ; A_{2}$, $A_{3}$ get color $(1,9)$; and $A_{4}, A_{5}, A_{6}, A_{7}$ get color $(2,9)$. No two vertices of the same color are disjoint or nested.

this coloring of $\operatorname{KG}(n)$ is proper. Suppose that $A$ and $B$ are different subsets that receive the same color $(k, a)$. We seek to show that $A$ and $B$ are neither disjoint nor is one contained in the other. Assume without loss of generality that $|A| \leqslant|B|$. As $a \in A \cap B$, these subsets are not disjoint. If $|A|=|B|$, then it is immediate that neither is contained in the other. If instead $|A|<|B|$, then $l(A)>l(B)$, so $A$ contains more elements greater than $a$ than $B$ does, while $2^{k+1}-2 l(B)>$ $2^{k+1}-2 l(A)$, so $B$ contains more elements less than $a$ than $A$ does. Therefore, neither of $A$ and $B$ is contained in the other in this case either. It follows that this coloring of $\mathrm{KG}(n)$ is proper and establishes the desired bound.

Proof of Theorem 1.1. Immediate from Theorems 2.3 and 2.5.

\section{Curves on planar surfaces}

In this section, we obtain a precise estimate on the chromatic number of the curve graph of a planar surface, and we determine its fractional chromatic number exactly. The methods of this section serve as a prototype for those appearing later on.

We assume that $n \geqslant 5$, so that $\mathcal{C}\left(\Sigma_{n}\right)$ contains edges. (When $n=4$, the definition of $\mathcal{C}\left(\Sigma_{4}\right)$ is usually altered so that edges consist of pairs of curves with minimal intersection number 2; see Section 8.) We establish the following more precise version of Theorem 1.2:

THEOREM 3.1. $\chi\left(\mathcal{C}\left(\Sigma_{n}\right)\right) \sim n \log n$ and $\chi_{f}\left(\mathcal{C}\left(\Sigma_{n}\right)\right)=\chi_{f}(\operatorname{KG}(n))-n$.

We split the proof of Theorem 3.1 into two easy lemmas. 
LEMMA 3.2. There exists a homomorphism $f: \mathcal{C}\left(\Sigma_{n}\right) \rightarrow \mathrm{KG}(n) \backslash \mathrm{KG}(n, 1)$.

Proof. A curve $c \in \mathcal{C}\left(\Sigma_{n}\right)$ induces a partition $f(c)$ of the components of $\partial \Sigma_{n}$ into two nonempty subsets according to which component of $\Sigma_{n} \backslash c$ they belong. Both subsets have size at least two, since $c$ is essential. If $c, d \in \mathcal{C}\left(\Sigma_{n}\right)$ are adjacent, then $\Sigma_{n} \backslash c \cup d$ consists of three components, each of which contains a component of $\partial \Sigma_{n}$. It follows that the partitions $f(c)$ and $f(d)$ are distinct and nested. Identifying the holes of $\Sigma_{n}$ with the underlying set of $\operatorname{KG}(n)$, the mapping $f$ defines the required homomorphism.

LEMma 3.3. There exists an embedding $c: \mathrm{CG}(n) \backslash \mathrm{CG}(n, 1) \hookrightarrow \mathcal{C}\left(\Sigma_{n}\right)$.

Proof. Embed an $n$-cycle in the 2 -sphere $\Sigma$. Label its vertices $p_{1}, \ldots, p_{n}$ cyclically and its edges $e_{i}=\left(p_{i}, p_{i+1}\right)$, indices $(\bmod n)$. Identify $\Sigma_{n}$ with the complement in $\Sigma$ of a small neighborhood of $\left\{p_{1}, \ldots, p_{n}\right\}$. For a vertex

$$
v=\{i, i+1, \ldots, i+k\} \in \mathrm{CG}(n) \backslash \mathrm{CG}(n, 1),
$$

let $c(v) \in \mathcal{C}\left(\Sigma_{n}\right)$ be the boundary of a regular neighborhood of

$$
e_{i} \cup \cdots \cup e_{i+k-1} \subset \Sigma \text {. }
$$

It is easy to see that the mapping $c$ defines the required embedding.

Proof of Theorem 3.1. The result follows from Theorem 1.1, Lemmas 3.2, 3.3 , and the monotonicity of the (fractional) chromatic number under homomorphisms. Note in addition that $\mathrm{KG}(n, 1) \subset \mathrm{KG}(n)$ and $\mathrm{CG}(n, 1) \subset \mathrm{CG}(n)$ are cliques of size $n$ adjacent to all other vertices; removing them from their supergraphs lowers the (fractional) chromatic numbers by $n$.

Observe that the exact value $\chi\left(\mathcal{C}\left(\Sigma_{5}\right)\right)=3$ follows as well from Lemmas 3.2 and 3.3, since both $\mathrm{KG}(5) \backslash \mathrm{KG}(5,1)=\mathrm{KG}(5,2)$ and $\mathrm{CG}(5,2)$ have chromatic number 3 .

The proof of Theorem 3.1 raises the question whether $\mathrm{KG}(n) \backslash \mathrm{KG}(n, 1)$ itself embeds in $\mathcal{C}\left(\Sigma_{n}\right)$. The following result shows that this is not the case for $n=5$.

Proposition 3.4. $\mathcal{C}\left(\Sigma_{5}\right)$ does not contain a subgraph isomorphic to $\mathrm{KG}(5,2)$.

Proof. Suppose, by way of contradiction, that there were a subgraph of $\mathcal{C}\left(\Sigma_{5}\right)$ isomorphic to $\operatorname{KG}(5,2)$. Restricting $f$ to the subgraph gives an endomorphism of $\mathrm{KG}(5,2)=\mathrm{KG}(5) \backslash \mathrm{KG}(5,1)$. Since any endomorphism of $\mathrm{KG}(n, k)$ is an automorphism [GR13, Theorem 7.9.1], it follows that the subgraph is the image 
of a section $s$ of $f$. Given a 2-element subset $\{i, j\}$, let $a_{i j}$ denote an arc, unique up to isotopy, with endpoints on the holes $\partial_{i}, \partial_{j}$ that is disjoint from $s(\{i, j\})$. Collapsing each hole to a point gives a drawing of $K_{5}$ on the sphere such that each pair of edges with disjoint endpoints do not cross. This contradicts a theorem of van Kampen and Flores, a strong form of the fact that $K_{5}$ is nonplanar (see [Mat03, Theorem 5.1.1]).

If one knew that all endomorphisms of $\mathrm{KG}(n) \backslash \mathrm{KG}(n, 1)$ are automorphisms, then the proof of Proposition 3.4 would readily adapt to show that $\mathrm{KG}(n) \backslash \mathrm{KG}(n, 1)$ is not a subgraph of $\mathcal{C}\left(\Sigma_{n}\right)$ for any $n \geqslant 5$. We presume this is the case, but a proof would take us too far afield.

\section{Separating curves}

The goal of this section is to prove Theorem 1.3:

THEOREM 1.3. If $S$ has Euler characteristic $-k<0$, then $\chi\left(\mathcal{C}_{\text {sep }}(S)\right) \sim k \log k$.

The proof strategy is similar to that of Theorem 3.1. However, we must replace the holes by a less obvious collection of points. They are provided by the following result, which draws inspiration from [BP07]:

THEOREM 4.1. If $S$ has Euler characteristic $-k<0$, then there exist a hyperbolic metric on $S$ and a subset $Q \subset S$ of $12 k$ points with the following two properties: (1) $S$ has totally geodesic boundary, and (2) if $F \subset S$ is a subsurface with totally geodesic boundary, then $Q \cap \partial F=\emptyset$ and

$$
-\chi(F)=\frac{1}{2 \pi} \operatorname{Area}(F)=\frac{1}{12}|Q \cap F| .
$$

Thus, the discrete uniform measure concentrated on the point set $Q$ is proportional to the standard area measure when restricted to subsurfaces with totally geodesic boundary.

We first derive Theorem 1.3 from Theorem 4.1.

Proof of Theorem 1.3. First, we establish the upper bound. Apply Theorem 4.1. A curve $c \in \mathcal{C}_{\text {sep }}(S)$ has a unique geodesic representative, which cuts $S$ into a pair of subsurfaces with totally geodesic boundary. We obtain a partition $f(c)$ of $Q$ into two parts according to the subsurfaces these points lie in. Identifying $Q$ with the underlying set of the Kneser graph $\mathrm{KG}(12 k)$, we obtain a map $f: \mathcal{C}_{\text {sep }}(S) \rightarrow$ $\mathrm{KG}(12 k)$. 


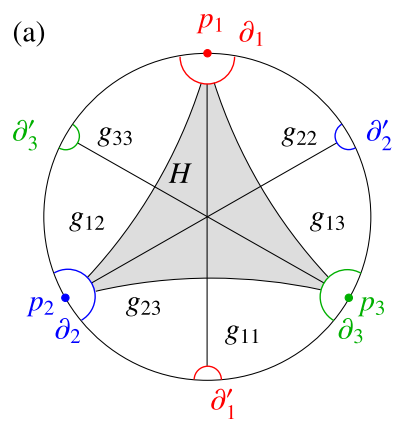

(b)

Figure 2. (a) A right-angled hexagon $H$ and distinguished geodesics in $\mathbb{D}^{2}$. (b) Two ideal quadrilaterals and three reflections of $H$.

We claim that $f$ is a homomorphism. If $c, d \in \mathcal{C}_{\text {sep }}(S)$ are adjacent, then they can be realized by disjoint, simple closed geodesics in $S$. The complement $S \backslash c \cup d$ consists of three components with totally geodesic boundary. Since the curves are essential and not parallel, each component has negative Euler characteristic. By Theorem 4.1, each subsurface contains points of $Q$, and it follows that $f(c)$ and $f(d)$ are nested. Therefore, $f$ is a homomorphism, as claimed. Theorem 2.5 and the monotonicity of the chromatic number under homomorphisms now lead to the stated upper bound.

For the lower bound, embed a planar surface $\Sigma=\Sigma_{g+b}$ into $S$ so that each component of $\partial \Sigma$ either bounds a subsurface $S_{1}^{1} \subset S$ or is a component of $\partial S$. Every essential curve in $\Sigma$ is essential and separating in $S$, and distinct curves in $\Sigma$ are distinct in $S$. The embedding $\Sigma \hookrightarrow S$ therefore induces an embedding $\mathcal{C}(\Sigma) \hookrightarrow \mathcal{C}_{\text {sep }}(S)$, and Theorem 3.1 gives the desired lower bound.

We now develop the proof of Theorem 4.1 through a sequence of lemmas in hyperbolic geometry. Roughly speaking, we apply a geometric limiting argument in which the curves in a pants decomposition get pinched. For background on hyperbolic geometry, see [Bus92, Ch. $1 \&$ 3] and [FM11, Sections 10.5-6].

We work in the Poincaré disk model $\mathbb{D}$ of the hyperbolic plane. Fix three ideal points $p_{1}, p_{2}, p_{3} \in \partial \overline{\mathbb{D}}$. Consider a right-angled geodesic hexagon $H \subset \mathbb{D}$ that is invariant under the symmetries of $\mathbb{D}$ permuting the points $p_{i}$. Extend the side of $H$ closest to $p_{i}$ to a complete geodesic $\partial_{i}$, and label its side with endpoints on $\partial_{i}$ and $\partial_{j}$ by $g_{i j}$. Let $\partial_{i}^{\prime}$ denote the reflection of $\partial_{i}$ across $g_{j k}$, where $\{i, j, k\}=\{1,2,3\}$, and let $g_{i i}$ denote the geodesic arc through the origin and perpendicular to $\partial_{i}$ with endpoints on $\partial_{i}$ and $\partial_{i}^{\prime}$. See Figure 2(a). 
By Poincaré's Polyhedron theorem, the reflections across the sides $g_{i j}$ of $H$ generate a subgroup $\Gamma<\operatorname{Isom}(\mathbb{D})$, and the images of $H$ under the action by $\Gamma$ tesselate a simply connected, convex region $\tilde{P} \subset \mathbb{D}$ (see [Bea83, Section 9.8]). For $1 \leqslant i \leqslant j \leqslant 3$, let $Q_{i j} \subset \tilde{P}$ denote the interior of the ideal quadrilateral determined by the boundary components of $\tilde{P}$ containing the endpoints of $g_{i j}$. See Figure 2(b).

LEMmA 4.2. The images of $H \cap Q_{i j}$ under the reflection group $\Gamma$ cover $Q_{i j}$.

Proof. Let $\sigma$ and $\sigma^{\prime}$ denote the sides of $\bar{Q}_{i j}$ interior to $\tilde{P}$, with $\sigma$ chosen to intersect $H$. Moving along $\sigma$ from the interior of $H$ to the ideal point of intersection between $\sigma$ and $\partial_{i}$, we encounter a sequence of edges $s_{1}, s_{2}, \ldots$ in the tesselation of $\tilde{P}$. The geodesic triangle $\Delta \subset Q_{i j}$ bounded by $\partial_{i}, \sigma$, and $s_{1}$ is tesselated by quadrilaterals $Q_{n}, n \geqslant 1$, where $Q_{n}$ is bounded by $s_{n}, \sigma, s_{n+1}$, and $\partial_{i}$. Let $r_{n}$ denote the reflection in the side $s_{n}$. For $n \geqslant 2$, observe that $r_{n}\left(Q_{n}\right)$ is the quadrilateral bounded by $s_{n}, r_{n}(\sigma), s_{n-1}$, and $\partial_{i}$. The geodesic $r_{n}(\sigma)$ passes through the point of intersection between $\sigma$ and $s_{n}$ and limits to the ideal point of intersection between $\partial_{i}$ and $\sigma^{\prime}$, and the portion of $r_{n}(\sigma)$ between these two points is contained in $Q_{i j}$ by convexity. It follows that $r_{n}\left(Q_{n}\right) \subset Q_{n-1}$ for all $n \geqslant 2$. Furthermore, $r_{1}\left(Q_{1}\right)$ is contained in $Q_{i j} \cap H$ by similar reasoning. It follows that the images of $Q_{i j} \cap H$ under the elements $r_{n} \cdots r_{1} \in \Gamma, n \geqslant 1$, cover $\Delta$. Let $k$ and $l$ denote the unique values so that $\{i, j\}$ and $\{k, l\}$ partition the set $\{1,2,3\}$. The region $Q_{i j}$ is the union of $Q_{i j} \cap H$, its image under the reflection across $g_{i j}$, and the images of $\Delta$ under the four elements generated by the reflections across the orthogonal sides $g_{i j}$ and $g_{k l}$. The statement of the Lemma now follows with the additional observation that $g_{i i} \Gamma g_{i i}=\Gamma$ for $i \in\{1,2,3\}$.

Let $\ell>0$ denote the common length of the sides of $H$ contained in the $\partial_{i}$. Observe that we obtain a one-parameter family of hexagons $H$ as above by varying the value $\ell \in \mathbb{R}^{+}$.

Lemma 4.3. As $\ell \rightarrow 0$, the complement of the $Q_{i j}$ in $H$ consists of six pairwise disjoint geodesic triangles whose angles limit to $(\pi / 2, \pi / 3,0)$.

Proof. The endpoints of $\partial_{i}$ are continuous functions of $\ell$, and they converge to $p_{i}$ as $\ell \rightarrow 0$. Therefore, as $\ell \rightarrow 0$, the $Q_{i j}$ converge to complete geodesics $G_{i j}$ and $H$ converges to the ideal triangle $\Delta$ with vertices $p_{1}, p_{2}, p_{3}$, where convergence is in the Hausdorff metric with respect to the Euclidean metric on $\mathbb{D}$. Observe that the complement of the $G_{i j}$ in $\Delta$ is the union of six $(\pi / 2, \pi / 3,0)$-triangles. 
Let $C=H \backslash \cup Q_{i j}$. From the preceding paragraph, it follows that $C$ has six connected components for small values of $\ell$. Notice that the $g_{i i}$ partition $H$ into six regions that each intersects exactly three of the $Q_{i j}$ and that each region contains a component of $C$. It follows that each component of $C$ is a geodesic triangle, and as $\ell \rightarrow 0$, its angles converge to those of its limiting triangle.

The subgroup $\Gamma_{0}=\Gamma \cap \operatorname{Isom}^{+}(\mathbb{D})$ has index two in $\Gamma$. The quotient $P=\tilde{P} / \Gamma_{0}$ is homeomorphic to a pair of pants $S_{0}^{3}$, and the quotient map $f: \tilde{P} \rightarrow P$ is its universal covering. The map $f$ endows $P$ with a hyperbolic structure in which each boundary component $f\left(\partial_{i}\right)$ is totally geodesic and has length $2 \ell$.

LEMMA 4.4. Every properly embedded, simple geodesic arc in $P$ is contained in the image under $f$ of the $Q_{i j}$.

Proof. Let $a_{i j} \subset P$ denote a properly embedded, simple geodesic arc that has one endpoint on $f\left(\partial_{i}\right)$ and the other on $f\left(\partial_{j}\right)$. The projection $f\left(g_{i j}\right)$ is a properly embedded, simple geodesic arc isotopic rel. $f\left(\partial_{i}\right), f\left(\partial_{j}\right)$ to $a_{i j}$. The isotopy from $f\left(g_{i j}\right)$ to $a_{i j}$ lifts to an isotopy from $g_{i j}$ to a lift $\tilde{a}_{i j} \subset \tilde{P}$. In particular, $\tilde{a}_{i j}$ is a geodesic arc with endpoints on the same boundary components of $\tilde{P}$ as $g_{i j}$. It follows that $\tilde{a}_{i j} \subset Q_{i j}$. Therefore, $a_{i j}=f\left(\tilde{a}_{i j}\right) \subset p\left(Q_{i j}\right)$, as required.

Proposition 4.5. As $\ell \rightarrow 0$, the complement of the properly embedded, simple geodesic arcs in $P$ contains 12 pairwise disjoint geodesic triangles whose angles limit to $(\pi / 2, \pi / 3,0)$.

Proof. Any reflection in $\Gamma \backslash \Gamma_{0}$ descends to the same order-two, orientationreversing isometry $r: P \rightarrow P$. The covering map $f$ is a homeomorphism from $H$ onto its image, and $f(H)$ and $r(f(H))$ tesselate $P$. The image of the $Q_{i j}$ under $f$ is the image of the $Q_{i j} \cap H$ under $p$ and its reflection under $r$, by Lemma 4.2. The 12 triangles are then the images under $f$, and their reflections under $r$, of the 6 triangles in Lemma 4.3. They lie in the complement of the properly embedded, simple geodesic arcs in $P$ by Lemma 4.4.

We are now ready to prove Theorem 4.1 .

Proof of Theorem 4.1. Choose a pants decomposition $S=P_{1} \cup \cdots \cup P_{k}$. Fix $\ell>0$ and place a hyperbolic structure on $S$ so that each boundary component of each $P_{i}$ has length $\ell$. Every simple closed geodesic in $S$ intersects each $P_{i}$ in a union of disjoint simple proper arcs or in a boundary component. By Proposition 4.5, as $\ell \rightarrow 0$, the set of simple closed geodesics in $S$ is disjoint from a set of $12 k$ pairwise disjoint geodesic triangles $\Delta_{1}, \ldots, \Delta_{12 k}, 12$ in each $P_{i}$, with the area of 
each $\Delta_{i}$ limiting to $\pi / 6$, the area of a $(\pi / 2, \pi / 3,0)$-triangle. It follows in turn that the complement of the $\Delta_{i}$ has area limiting to 0 as $\operatorname{Area}(S)=2 \pi k$. Thus, for $\ell$ chosen suitably small, the area of a subsurface $F \subset S$ with totally geodesic boundary must equal to within 0.5 , say, of $\pi / 6$ times the number of the $\Delta_{i}$ that it contains. On the other hand, $\operatorname{Area}(F)=-2 \pi \chi(F)$ by the Gauss-Bonnet formula, which is an integral multiple of $\pi / 6$. It follows that $\operatorname{Area}(F)$ equals to exactly $\pi / 6$ times the number of the $\Delta_{i}$ that it contains. To finish the proof, let $Q$ consist of a single point from each $\Delta_{i}$.

\section{The graph of homologous curves}

The goal of this section is to study the chromatic number of the subgraph of $\mathcal{C}(S)$ induced on curves that can be oriented to represent a fixed nonzero homology class. The main theme is that this subgraph has a unique minimal coloring, which can be expressed in terms of genera of immersed surfaces. Section 5.1 treats the case of coloring a closed surface. Section 5.2 recasts this coloring in terms of domains, which is critical for the material of Section 6. Section 5.3 applies the coloring to establish the upper bound on $\chi\left(\mathcal{C}\left(S_{g}\right)\right)$ stated in Theorem 1.5. Section 5.4 establishes the uniqueness of the minimal coloring in the case of a closed surface. Finally, Section 5.5 treats the case of a surface with boundary.

5.1. The chromatic number of $\mathcal{C}_{v}\left(S_{g}\right)$. Let $v \in H_{1}\left(S_{g} ; \mathbb{Z}\right)$ denote a nonzero primitive element, and let $\mathcal{C}_{v}(S)$ denote the subgraph of $\mathcal{C}(S)$ induced on the curves that can be oriented to represent $v$. The main result of this subsection is:

TheOREM 5.1. $\chi\left(\mathcal{C}_{v}\left(S_{g}\right)\right)=\omega\left(\mathcal{C}_{v}\left(S_{g}\right)\right)=g-1$.

REMARK 5.2. Although its clique number and chromatic number are equal, the graph $\mathcal{C}_{v}\left(S_{g}\right)$ is typically not perfect: not all of its induced subgraphs have this property. Figure 3(a) displays an induced five-cycle in $\mathcal{C}_{v}\left(S_{g}\right)$ for $g \geqslant 6$.

The idea behind the proof of Theorem 5.1 is simple, but it takes some work to establish, which we bundle into a few Lemmas in covering space theory. Suppose that $c$ and $d$ are homologous, oriented, simple closed curves in $S_{g}$. Define an immersion (of genus $h$ ) from $c$ to $d$ to be an orientation-preserving immersion $i$ from an oriented surface $S_{h}^{2}$ to $S_{g}$ that maps one boundary component, oriented as $\partial S_{h}^{2}$, injectively to $-c$ and the other to $d$. 


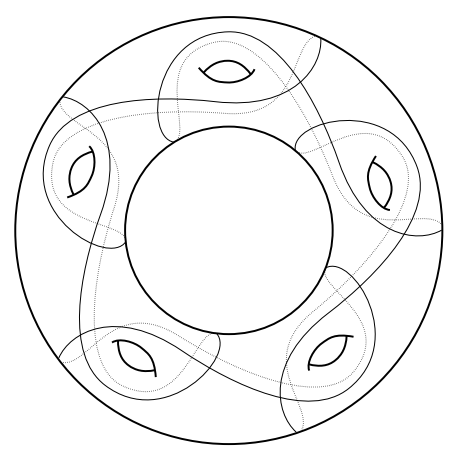

(a) An induced five-cycle.

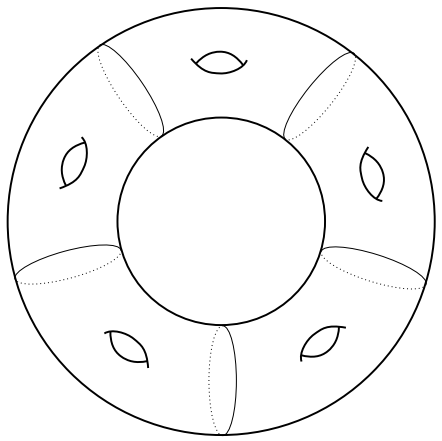

(b) A maximal clique.

Figure 3. Subgraphs of $\mathcal{C}_{v}\left(S_{6}\right)$.

LEMMA 5.3. There exists an immersion between any pair of homologous, oriented, nonseparating simple closed curves on $S_{g}$.

The proof of Lemma 5.3 relies on a construction of the infinite cyclic cover $\left(S, p_{v}\right)$ corresponding to the epimorphism $\iota(v,-): H_{1}\left(S_{g} ; \mathbb{Z}\right) \rightarrow \mathbb{Z}$, where $v \in H_{1}\left(S_{g} ; \mathbb{Z}\right)$ denotes a nonzero primitive class. It is reminiscent of, but simpler than, the construction of the infinite cyclic cover of a knot exterior. Let $c$ be an oriented simple closed curve representing $v$. Cut open $S_{g}$ along an open regular neighborhood of $c$ to form the surface $S \backslash N(c) \approx S_{g-1}^{2}$, whose oriented boundary consists of two components, labeled $c^{+}$and $c^{-}$. Form $(S \backslash N(c)) \times \mathbb{Z}$, and identify $c^{+} \times\{n\}$ with $c^{-} \times\{n+1\}$ by an orientation-reversing homeomorphism for all $n \in \mathbb{Z}$. The resulting quotient space is $S_{\infty}$, and it comes equipped with a covering map $p_{c}$.

LEMMA 5.4. The covering spaces $\left(S_{\infty}, p_{c}\right)$ and $\left(S, p_{v}\right)$ are isomorphic.

Proof. By construction, $\left(S_{\infty}, p_{c}\right)$ is a regular cover of $S_{g}$ with deck transformation group $\mathbb{Z}$. Consequently, it corresponds to some epimorphism $\varphi: H_{1}\left(S_{g} ; \mathbb{Z}\right) \rightarrow \mathbb{Z}$. To identify $\varphi$, complete $c$ to a geometric symplectic basis $c=a_{1}, b_{1}, \ldots, a_{g}, b_{g}$ for $S_{g}$, that is a collection of simple closed curves such that the only pairwise intersections between these curves are a single transverse point of intersection between $a_{i}$ and $b_{i}$ for each $i=1, \ldots, g$. By construction, each of these basis curves lifts to a simple closed curve in $S_{\infty}$ with the exception of $b_{1}$. Therefore, $\varphi$ vanishes precisely on the subspace of $H_{1}\left(S_{g} ; \mathbb{Z}\right)$ spanned by $\left[a_{1}\right],\left[a_{2}\right],\left[b_{2}\right], \ldots$, $\left[a_{g}\right],\left[b_{g}\right]$. This subspace is $\operatorname{ker} \iota(v,-)$, so $\varphi=\iota(v,-)$, and the statement of the lemma follows. 
Proof of Lemma 5.3. Suppose that $c$ and $d$ are simple closed curves that represent the class $v \in H_{1}\left(S_{g} ; \mathbb{Z}\right), v \neq 0$. Let $\left(S_{\infty}, p_{v}\right)$ denote the corresponding infinite cyclic cover. Fix a lift $\tilde{c}$ to $S_{\infty}$ by Lemma 5.4, and let $t$ denote a generator of the deck transformation group. By the construction of $\left(S_{\infty}, p_{v}\right)$ justified in Lemma 5.4, the lift $\widetilde{c}$ separates $S_{\infty}$ into two components $S_{\infty}^{ \pm}(\widetilde{c})$, labeled so that $t \cdot S_{\infty}^{+}(\widetilde{c}) \subset S_{\infty}^{+}(\widetilde{c})$. Since $[d]=v$, the same remarks apply to $d$ as well. In particular, we can choose a lift of $d$ to a simple closed curve $\widetilde{d}$ contained in $S_{\infty}^{+}(\widetilde{c})$; in particular, $\widetilde{c} \cap \widetilde{d}=\emptyset$. The curve $\widetilde{d}$ separates $S_{\infty}$ into two components $S_{\infty}^{ \pm}(\widetilde{d})$, labeled so that $t \cdot S_{\infty}^{+}(\widetilde{d}) \subset S_{\infty}^{+}(\widetilde{d})$. The lifts $-\widetilde{c}$ and $\widetilde{d}$ cobound the compact subsurface $T=S_{\infty}^{+}(\widetilde{c}) \cap S_{\infty}^{-}(\widetilde{d})$, and $T \approx S_{h}^{2}$ for some $h \geqslant 0$. The desired immersion $i$ is the restriction of the covering map $p_{v}$ to $T$.

LEMMA 5.5. Any two immersions between a pair of nonseparating simple closed curves on $S_{g}$ have the same genus $(\bmod g-1)$, and this value depends only on the isotopy classes of the curves.

Proof. Suppose that $c$ and $d$ are homologous simple closed curves on $S_{g}$ and we have an immersion $i_{1}: S_{h_{1}}^{2} \rightarrow S_{g}$ from $c$ to $d$. There exists an immersion $i_{2}: S_{h_{2}}^{2} \rightarrow S_{g}$ from $d$ to $c$ as well by Lemma 5.3. Select an orientation-reversing diffeomorphism $\varphi: \partial S_{h_{1}}^{2} \rightarrow \partial S_{h_{2}}^{2}$ so that $i_{2}=i_{1} \circ \varphi$ on $\partial S_{h_{2}}^{2}$. Glue the two surfaces using $\varphi$ to obtain an immersion $i: S_{h_{1}+h_{2}+1} \rightarrow S_{g}$. As the immersion is a covering map, the Euler characteristic of the base divides that of the cover, leading to the congruence $h_{1}+h_{2} \equiv 0(\bmod g-1)$. In particular, the genus of any immersion from $c$ to $d$ is congruent to the fixed value $-h_{2}(\bmod g-1)$. The fact that this value depends only on the isotopy classes of $c$ and $d$ follows by an easy argument using the isotopy extension principle.

Proof of Theorem 5.1. We build a clique of size $g-1$ in $\mathcal{C}_{v}\left(S_{g}\right)$ by cyclically gluing together $g-1$ copies of the surface $S_{1}^{2}$ along their boundaries and taking the images of the boundary curves. See Figure 3(b).

We obtain an optimal coloring $f: \mathcal{C}_{v}\left(S_{g}\right) \rightarrow \mathbb{Z} /(g-1) \mathbb{Z}$ as follows. Fix an oriented simple closed curve $c \subset S$ representing the class $v$. Given an oriented simple closed curve $d \subset S$ that also represents $v$, there exists an immersion from $c$ to $d$, by Lemma 5.3. Its genus $h(\bmod g-1)$ depends only on the isotopy type of $d$, by Lemma 5.5. We may therefore unambiguously define $f(d) \equiv h$ $(\bmod g-1)$.

To show that the coloring $f$ is proper, suppose that $d_{1}$ and $d_{2}$ are disjoint simple closed curves that represent the class $v$. Thus, there exists a subsurface $T \subset S$ with oriented boundary $d_{2}-d_{1}$ and genus $0<t<g-1$. Stacking $T$ onto an immersion $i: S_{h}^{2} \rightarrow S$ from $c$ to $d_{1}$ gives an immersion from $c$ to $d_{2}$ of genus 
$h+t \not \equiv h(\bmod g-1)$. It follows that $f\left(d_{1}\right) \neq f\left(d_{2}\right)$, so the coloring is proper, as desired.

5.2. Domains. The coloring $f$ described in the proof of Theorem 5.1 admits an alternate description in terms of domains on $S$ that we will make use of in Section 6 (see Lemmas 6.1 and 6.2). Domains and their combinatorial Euler measures recur throughout Heegaard Floer homology; see [Sar11, Section 2] for a quick, thorough treatment. In our setting, this description allows us to compute $f$ directly on $S$, without passing to the cover $S_{\infty}$.

As in the proof of Theorem 5.1, fix an oriented simple closed curve $c$ that represents $v$, and choose another oriented simple closed curve $d$ that also represents $v$. Position $c$ and $d$ to meet transversely. The complement $S-c-d$ consists of a number of connected components whose closures are called regions. The classes of the regions form a basis for $H_{2}(S, c \cup d$; $\mathbb{Z})$. An element of this group is called a domain on $(S, c \cup d)$. The neighborhood of each intersection point in $c \cap d$ contains four corners. For each region $R$, let $e(R)$ denote the Euler characteristic of its interior and $c(R)$ its number of corners. We define the combinatorial Euler measure of a region $R$ by $m(R)=e(R)-c(R) / 4$. Its definition extends to domains by linearity. Since $c$ and $d$ are homologous, there exists a domain $D$ with $\partial D=d-c$. The domain is well defined up to multiples of $[S]=\sum_{R}[R]$. It follows that the value $m(D)$ is well-defined modulo $m([S])=e(S)=-2(g-1)$. Define $f^{\prime}(d)=-\frac{1}{2} m(D)(\bmod g-1)$.

Proposition 5.6. The maps $f$ and $f^{\prime}$ are equal.

Proof. Choose a curve $d$ representing the class $v$. Choose disjoint lifts $\widetilde{c}$ of $c$ and $\widetilde{d}$ of $d$ to $S_{\infty}$, and let $\Sigma \subset S_{\infty}$ be the compact subsurface with $\partial \Sigma=\widetilde{d}-\widetilde{c}$, as in the proof of Lemma 5.3. The projection $p_{v}(\Sigma)$ gives a domain in $(S, c \cup d)$ with boundary $d-c$. We have $f(d) \equiv g(\Sigma)=-\frac{1}{2} m(\Sigma)=-\frac{1}{2} m\left(p_{v}(\Sigma)\right) \equiv f^{\prime}(d)$ (mod $g-1)$, using the additivity of $m$ in the third equality.

As a corollary to Proposition 5.6, the definition of $f^{\prime}$ descends to isotopy classes of curves. We could instead establish this fact directly from the definition of $f^{\prime}$ and an application of the bigon criterion. We could also base a proof of Theorem 5.1 on the definition of $f^{\prime}$ instead of $f$. In particular, $f^{\prime}$ is a proper coloring, although it is less immediate from its definition that it only takes integer values and not just half-integer values, which is required to see that it is a proper $(g-1)$-coloring and not just a proper $2(g-1)$-coloring.

REMARK 5.7. Domains and their combinatorial Euler measures behave well under refinement, an observation we shall require in the proof of Lemma 6.1. 


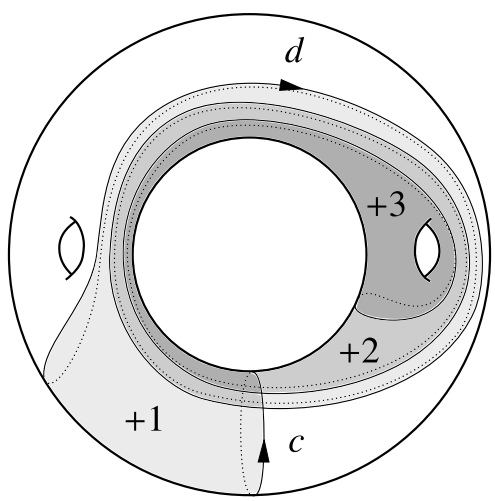

Figure 4. The sum of the shaded regions, weighted according to labels, form a domain whose boundary is $d-c$. As its combinatorial Euler measure is $-6 \equiv 0$ $(\bmod 2), c$ and $d$ have the same color.

Suppose that $\Gamma \subset S$ is a union of simple closed curves that contains $c$ and $d$, meet transversely, and only have double points of intersection. We can define domains on $(S, \Gamma)$ and their combinatorial Euler measures just as above. Suppose that $D^{\prime}=\sum n_{i}^{\prime}\left[R_{i}^{\prime}\right]$ is a domain on $(S, \Gamma)$ with $\partial D=d-c$. Each region $R_{j}$ of $S-c \cup d$ is a union of regions $R_{i}^{\prime}$ of $S-\Gamma$ for $i$ in some index set $I_{j}$, and the coefficients $n_{i}^{\prime}, i \in I_{j}$, are equal to some constant value $n_{j}$. We thereby obtain a domain $D=\sum n_{j}\left[R_{j}\right]$ on $(S, c \cup d)$ with $\partial D=d-c$. Linearity of the combinatorial Euler measure under taking unions ensures that $m(D)=m\left(D^{\prime}\right)$. For this reason, given homologous curves $c$ and $d$ belonging to $\Gamma \subset S$, we get a single welldefined value $m(D)$ (mod $g-1)$ for a domain $D$ from $c$ to $d$, independent of the other curves in $\Gamma$. Furthermore, we may add domains corresponding to different sets of curves $\Gamma_{1}$ and $\Gamma_{2}$ on $S$ by passing to the union $\Gamma_{1} \cup \Gamma_{2}$.

REMARK 5.8. In a slightly different guise, the map $f^{\prime}$ was considered by Irmer: it is twice the signed length from the chosen basepoint $c \in \mathcal{C}_{v}(S)$ [Irm15, Lemma 3]. We note that Irmer's description of the computation of $f^{\prime}(d)$ requires a path from $c$ to $d$ in a certain subgraph of $\mathcal{C}_{v}\left(S_{g}\right)$, whereas ours requires only a realization of $c$ and $d$ and the domain they cobound. For instance, for the curves $c$ and $d$ pictured in Figure $4, f^{\prime}(d) \equiv 0$. In particular, $c$ and $d$ are curves of the same color.

REMARK 5.9. Given the difference between the orders of growth of the chromatic numbers of $\mathcal{C}_{\text {sep }}(S)$ and $\mathcal{C}_{v}(S), v \neq 0$, the reader may wonder how the $(g-1)$-coloring of $\mathcal{C}_{v}(S)$ fails for $v=0$. For example, even when $g=3$, we 
have $\omega\left(\mathcal{C}_{\text {sep }}\left(S_{3}\right)\right)=3>2=\chi\left(\mathcal{C}_{v}\left(S_{3}\right)\right), v \neq 0$. The issue is explained in part by the fact that Lemma 5.3 fails in this setting: there does not exist a consistent way to orient the separating curves so that there exists an immersion between any two, as the reader may check on a maximum clique in $\mathcal{C}_{\text {sep }}\left(S_{3}\right)$. Moreover, there exist domains of Euler measure 0 cobounded by disjoint oriented separating curves: take the image of an injective immersion $S_{1}^{1} \sqcup S_{1}^{1} \rightarrow S_{g}, g \geqslant 3$, that reverses orientation on one component.

5.3. The upper bound $\chi\left(\mathcal{C}\left(S_{g}\right)\right) \lesssim g \cdot 4^{g}$. In this subsection, we use Theorem 5.1 to obtain the upper bound in Theorem 1.5. First, we extend Theorem 5.1 to the graph $\mathcal{C}_{\bar{v}}\left(S_{g}\right)$ induced on curves that can be oriented to represent a fixed nonzero primitive homology class $\bar{v} \in H_{1}\left(S_{g} ; \mathbb{Z} / m \mathbb{Z}\right)$, where $m>1$ is a positive integer.

Proposition 5.10. $\chi\left(\mathcal{C}_{\bar{v}}\left(S_{g}\right)\right)=g-1$.

Proof. Let $V \subset H_{1}\left(S_{g} ; \mathbb{Z}\right)$ denote the set of elements that reduce to $\bar{v}(\bmod m)$. If $c$ and $d$ are disjoint representatives of $\bar{v}$, then they cobound a subsurface of $S_{g}$, so they represent the same element $v \in V$. (Recall that an unoriented curve represents $v$ if some orientation of it does.) It follows that $\mathcal{C}_{\bar{v}}\left(S_{g}\right)$ is the disjoint union of the induced subgraphs $\mathcal{C}_{v}\left(S_{g}\right), v \in V$. The $(g-1)$-coloring $\mathcal{C}_{v}\left(S_{g}\right) \rightarrow \mathbb{Z} /(g-1) \mathbb{Z}$ described in the proof of Theorem 5.1 depends on a choice of representative $c_{v} \in \mathcal{C}_{v}\left(S_{g}\right)$ for each $v \in V$. A priori, the simultaneous existence of these representatives depends on the axiom of (countable) choice. However, we can remove this dependence as follows. Meeks and Patrusky establish an algorithm to produce a curve $c_{v}$ representing the class $v$ [MP78, Section 1] (see also [FM11, Proposition 6.2]). The algorithm depends on a fixed choice of geometric symplectic basis for $S_{g}$ and finitely many paths in $S_{g}$, and these choices are independent of $v$. Taking the representative $c_{v}$ of $v$ output by the algorithm for all $v \in V$ produces the desired explicit set of representatives. Their existence produces a simultaneous proper $(g-1)$-coloring of all $\mathcal{C}_{v}\left(S_{g}\right), v \in V$, whence the desired proper $(g-1)$-coloring of $\mathcal{C}_{\bar{v}}\left(S_{g}\right)$.

Recall the subgraph $\mathcal{N}\left(S_{g}\right)$ of $\mathcal{C}\left(S_{g}\right)$ induced on the nonseparating curves.

Corollary 5.11. $\chi\left(\mathcal{N}\left(S_{g}\right)\right) \leqslant(g-1) \cdot\left(2^{2 g}-1\right)$.

Proof. Give each subcomplex $\mathcal{C}_{\bar{v}}\left(S_{g}\right), \bar{v} \in H_{1}\left(S_{g} ; \mathbb{Z} / 2 \mathbb{Z}\right) \backslash\{0\}$, a proper $(g-1)$ coloring by Proposition 5.10, using a different color pallette for each. As there are $2^{2 g}-1$ elements in $H_{1}\left(S_{g} ; \mathbb{Z} / 2 \mathbb{Z}\right) \backslash\{0\}$, the stated bound follows. 
Recall Theorem 1.5:

THEOREM 1.5. $g \cdot \log g \lesssim \chi\left(\mathcal{C}\left(S_{g}\right)\right) \lesssim g \cdot 4^{g}$

Proof of Theorem 1.5. Immediate from Theorem 1.3 and Corollary 5.11.

5.4. Unique colorability of $\mathcal{C}_{v}(S)$. A graph is uniquely $k$-colorable if it admits a proper $k$-coloring and any two proper $k$-colorings are related by a bijection between the color sets. In other words, the graph admits a unique partition into $k$ independent sets. We establish the following complement to Theorem 5.1:

THEOREM 5.12. $C_{v}\left(S_{g}\right)$ is uniquely $(g-1)$-colorable.

Let $S=S_{g}^{b}, g \geqslant 1, b \in\{0,2\}$. If $b=0$, let $v \in H_{1}(S ; \mathbb{Z})$ denote a primitive, nonzero class, and if $b=2$, let $v$ denote the peripheral class. Recall the maximal clique graph $\mathcal{K}(\mathcal{C})$ of an arbitrary finite-dimensional simplicial complex $\mathcal{C}$, whose vertices consist of the maximal simplices in $\mathcal{C}$, and where two maximal simplices are adjacent if they meet in a codimension- 1 face.

Proposition 5.13. $\mathcal{K}\left(\mathcal{C}_{v}(S)\right)$ is connected.

Proof. First, suppose that $b=2$. We show that $\mathcal{K}\left(\mathcal{C}_{v}(S)\right)$ is connected in this case using Putman's technique [Put08, Lemma 2.1]. Since $v$ is the peripheral class, the mapping class group $\operatorname{PMod}(S)$ acts on $\mathcal{K}\left(\mathcal{C}_{v}(S)\right)$, and by the change of coordinates principle (see [FM11, Section 1.3]), it does so transitively. Next, consider Figure 5. The Dehn twists about the red curves shown there constitute the Humphries generating set for $\operatorname{PMod}\left(S_{g-1}^{2}\right)$ [FM11, Figure 4.10]. The blue curves shown there constitute a maximal simplex $K$ in $\mathcal{C}_{v}(S)$. Observe that each red curve meets at most one blue curve, and the algebraic intersection number between any red and blue curve is 0 . It follows that a Humphries generator either preserves $K$ or moves it to another maximal simplex that meets $K$ in a codimension-1 face. Therefore, [Put08, Lemma 2.1] applies to show that $\mathcal{K}\left(\mathcal{C}_{v}(S)\right)$ is connected.

Next, suppose that $b=0$. If $g=1$, then $\mathcal{C}_{v}(S)$ is a single vertex. If $g=2$, then $\mathcal{C}_{v}(S)$ has no edges, so the maximal simplices are its vertices. Any two vertices meet in a codimension-1 (empty) face, so $\mathcal{K}\left(\mathcal{C}_{v}(S)\right)$ is an infinite complete graph on $\mathcal{C}_{v}(S)$. The desired result is trivial in either case. Suppose then that $g \geqslant 3$. Choose a pair of maximal simplices $K$ and $K^{\prime}$ in $\mathcal{C}_{v}(S)$, a curve $c$ in $K$, and a curve $c^{\prime}$ in $K^{\prime}$. As $\mathcal{C}_{v}(S)$ is connected [Put08, Theorem 1.9], there exists a path $c=a_{1}$, $a_{2}, \ldots, a_{n}=c^{\prime}$ in $\mathcal{C}_{v}(S)$ connecting $c$ and $c^{\prime}$. Thus, there exists a maximal simplex 


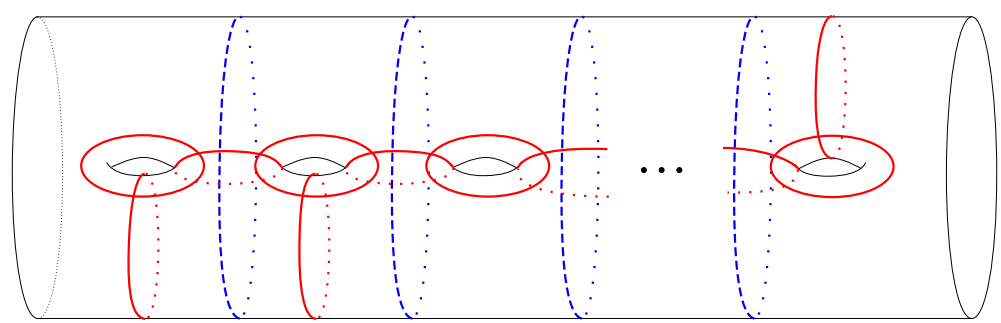

Figure 5. The surface $S_{g}^{2}$. The Dehn twists about the solid red curves are Humphries generators for $\operatorname{PMod}\left(S_{g}^{2}\right)$. The dashed blue curves comprise a maximal simplex in $\mathcal{C}_{v}\left(S_{g}^{2}\right)$, where $v$ denotes the peripheral homology class.

$K_{i}$ containing $a_{i}$ and $a_{i+1}$ for all $i=1, \ldots, n-1$. Additionally, set $K_{0}=K$ and $K_{n}=K^{\prime}$. For all $i$, the link $\operatorname{Lk}\left(a_{i}\right)$ is isomorphic to $\mathcal{C}_{v^{\prime}}\left(S_{g-1}^{2}\right)$, where $v^{\prime}$ denotes the peripheral class. Therefore, there exists a sequence of maximal cliques in $\operatorname{Lk}\left(a_{i}\right)$, beginning with $K_{i-1} \cap \operatorname{Lk}\left(a_{i}\right)$ and ending with $K_{i} \cap \operatorname{Lk}\left(a_{i}\right)$, such that any two in sequence meet in a codimension- 1 face. Taking the joins of these simplices with $\left\{a_{i}\right\}$ results in a path from $K_{i-1}$ to $K_{i}$ in $\mathcal{K}\left(\mathcal{C}_{v}(S)\right)$. The concatenation of these paths is a path from $K$ to $K^{\prime}$ in $\mathcal{K}\left(\mathcal{C}_{v}(S)\right)$. Therefore, $\mathcal{K}\left(\mathcal{C}_{v}(S)\right)$ is connected.

A simplicial complex is pure of dimension $l$ if every simplex is contained in an $l$-simplex. The proof of the following result is straightforward.

LEMMA 5.14. If a simplicial complex $\mathcal{C}$ is pure of dimension $k-1$ and properly $k$-colorable, and $\mathcal{K}(\mathcal{C})$ is connected, then $\mathcal{C}$ is uniquely $k$-colorable.

Proof of Theorem 5.12. Theorem 5.1 shows that $\chi\left(C_{v}\left(S_{g}\right)\right)=g-1$. Proposition 5.13 shows that $\mathcal{K}\left(\mathcal{C}_{v}(S)\right)$ is connected. Lemma 5.14 now gives the desired result.

5.5. Surfaces with boundary. We now turn to the case of a surface with boundary $S=S_{g}^{b}, b \geqslant 1$. We now permit $v=0$ in defining $\mathcal{C}_{v}(S)$. More cases arise due to the distinction of whether a separating homology class is null-homologous, peripheral, or not. As with a closed surface, we find a qualitative difference between $v=0$ and $v \neq 0$. However, for each type of $v$, the growth of $\chi\left(\mathcal{C}_{v}(S)\right)$ is governed solely by $g$ and not by $b$ (and hence not the Euler characteristic, in contrast with Theorem 1.3).

THEOREM 5.15. $\chi\left(\mathcal{C}_{0}\left(S_{g}^{b}\right)\right) \sim g \cdot \log g$. 
Proof. Given $c \in \mathcal{C}_{0}(S)$, let $F(c)$ denote the component of $S \backslash c$ containing $\partial S$. Let $G_{0}(S)$ denote the subgraph induced on the curves $c$ for which $F(c)$ has genus 0 , and let $G_{+}(S)$ denote the subgraph on curves for which $F(c)$ has positive genus. Note that $G_{0}(S)$ is an independent set in $\mathcal{C}_{0}(S)$, and it consists of precisely the curves in $\mathcal{C}_{0}(S)$ whose images are inessential under the inclusion $i: S \hookrightarrow S_{g}$ obtained by capping off each component of $\partial S$ by a disk. It follows that $i$ induces a map $f: G_{+}(S) \rightarrow \mathcal{C}_{\text {sep }}\left(S_{g}\right)$. If $c$ and $d$ span an edge in $G_{+}(S)$, then $S \backslash c \cup d$ consists of three components of positive genus, and the same is true of $S_{g}$ > $f(c) \cup f(d)$. It follows that $f$ is a homomorphism. (However, $f$ is typically not injective: distinct curves in $G_{+}(S)$ related by a Dehn twist about a curve in $G_{0}(S)$ will have the same image under $f$.) Since the chromatic number is monotone under homomorphisms, it follows that $\chi\left(G_{+}(S)\right) \leqslant \chi\left(\mathcal{C}_{\text {sep }}\left(S_{g}\right)\right) \sim g \cdot \log g$, and using one more color on $G_{0}(S)$ gives the required upper bound on $\chi\left(\mathcal{C}_{0}(S)\right)$.

For the lower bound, embed a planar surface $\Sigma_{g+1} \hookrightarrow S$ so that one component of $\partial \Sigma_{g+1}$ is in $G_{0}(S)$ and all of the others bound disjoint subsurfaces $S_{1}^{1} \subset S$. The inclusion induces an embedding $\mathcal{C}\left(\Sigma_{g+1}\right) \hookrightarrow \mathcal{C}_{0}(S)$, and Theorem 3.1 supplies the lower bound.

THEOREM 5.16. For $v \neq 0, b \geqslant 1, \mathcal{C}_{v}\left(S_{g}^{b}\right)$ is uniquely $t$-colorable, where

$$
t= \begin{cases}g-1 & \text { if } v \text { is peripheral and } b=2 \\ g & \text { if } v \text { is nonseparating, or if } v \text { is peripheral and } b>2 \\ g+1 & \text { if } v \text { is nonperipheral and separating. }\end{cases}
$$

Moreover, $t=\chi\left(\mathcal{C}_{v}\left(S_{g}^{b}\right)\right)=\omega\left(\mathcal{C}_{v}\left(S_{g}^{b}\right)\right)$.

Proof. First, we describe the optimal coloring. For the case of a separating class $v$, fix a boundary component $\partial_{0} \subset \partial S$, and color a curve $c \in C_{v}(S)$ by the genus of the subsurface of $S \backslash c$ containing $\partial_{0}$. For the case of a nonseparating class $v$, we adapt the coloring described in Theorem 5.1. Now the lifts $\widetilde{c}$ and $\widetilde{d}$ cobound a compact subsurface $\widetilde{T} \subset S_{\infty}$ with some components of $\partial S_{\infty}$. We color $d$ by adding the genus of $\widetilde{T}$ to the number of preimages of $\partial_{0}$ in $\partial \widetilde{T}$ and reducing (mod $g$ ). In each case, it is straightforward to check that the coloring so described is proper, uses the stated number of colors, and that there exists a clique of the stated size. Figure 6 displays a maximal clique in the last case. The proof of unique colorability proceeds along the lines of Section 5.4 with minor changes, using the generating set for $\operatorname{PMod}\left(S_{g}^{b}\right)$ displayed in [FM11, Figure 4.10]. 


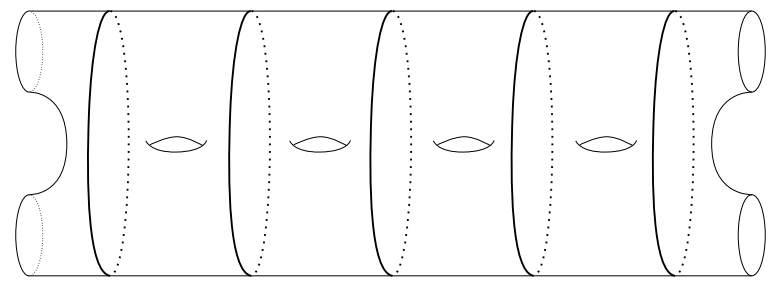

Figure 6. A $(g+1)$-clique in $\mathcal{C}_{v}\left(S_{g}^{b}\right), v$ a nonperipheral separating class.

\section{Permuting the colors and the Johnson homomorphism}

Throughout this section, we restrict to the case of a closed surface $S=S_{g}$. Because $\mathcal{C}_{v}(S)$ is uniquely $(g-1)$-colorable, any automorphism of it must permute the colors in a $(g-1)$-coloring. We therefore obtain a homomorphism from the automorphism group $\operatorname{Aut}\left(\mathcal{C}_{v}(S)\right)$ to the symmetry group of the colors. In this section, we investigate the restriction of this homomorphism to the Torelli group, which arises as a subgroup of $\operatorname{Aut}\left(\mathcal{C}_{v}(S)\right)$. As a result, we find a precise relationship between the permutation of the colors and the well-studied Johnson homomorphism. For background and references on the Torelli group and the Johnson homomorphism, see [FM11, Sections 6.5-6.6] and [Put18].

6.1. The action of the Torelli group on the color classes. Set $H=H_{1}(S ; \mathbb{Z})$, let $v \in H$ denote a primitive, nonzero class, and let $\mathcal{I}<\operatorname{Mod}(S)$ denote the Torelli group, the mapping classes that act trivially on $H$. Because $\mathcal{I}$ acts by graph automorphisms of $\mathcal{C}_{v}(S)$, Theorem 5.12 implies that we obtain a homomorphism $\chi_{v}: \mathcal{I} \rightarrow \operatorname{Sym}(g-1)$ that records the permutation of the colors in a $(g-1)$ coloring of $\mathcal{C}_{v}(S)$. Fix an orientation-preserving homeomorphism $\phi$ representing a mapping class in $\mathcal{I}$. Note that the coloring $f: \mathcal{C}_{v}(S) \rightarrow \mathbb{Z} /(g-1) \mathbb{Z}$ produced in Theorem 5.1 required the choice of a fixed oriented curve $c \in \mathcal{C}_{v}(S)$. The following result shows that the permutation induced by $\phi$ does not depend on this choice, and that it permutes the colors cyclically:

LEMMA 6.1. Let $d$ be any oriented simple closed curve representing $v \in H$, and let $C(d, \phi \cdot d)$ be a domain satisfying $\partial C(d, \phi \cdot d)=\phi \cdot d-d$. Then the permutation $\chi_{v}(\phi)$ shifts every color by $-\frac{1}{2} m(C(d, \phi \cdot d))(\bmod g-1)$.

Recall that $m(C)$ denotes the combinatorial Euler measure of the domain $C$, introduced in Section 5.2. In the following proof, we rely on the discussion in Remark 5.7 in order to treat domains in the presence of many curves on a surface. 
Proof. Fix an oriented curve $c \in \mathcal{C}_{v}(S)$. By Proposition 5.6, the coloring $f$ : $\mathcal{C}_{v}(S) \rightarrow \mathbb{Z} /(g-1) \mathbb{Z}$ is given by

$$
f(\gamma)=-\frac{1}{2} m(C(c, \gamma)) \quad(\bmod g-1),
$$

for any curve $\gamma$ representing $v$ and any domain $C(c, \gamma)$ satisfying $\partial C(c, \gamma)=$ $\gamma-c$. Let $C(\gamma, \phi \cdot \gamma)$ be a domain with boundary $\phi \cdot \gamma-\gamma$, and note that $C(c, \gamma)+C(\gamma, \phi \cdot \gamma)$ is a domain with boundary $\phi \cdot \gamma-c$. Since $m$ is additive, it follows that

$$
f(\phi \cdot \gamma)-f(\gamma)=-\frac{1}{2} m(C(\gamma, \phi \cdot \gamma)) \quad(\bmod g-1) .
$$

We wish to see that the right side equals $-\frac{1}{2} m(C(d, \phi \cdot d))(\bmod g-1)$, independent of $\gamma$. Choose a domain $C(d, \gamma)$ with boundary $\gamma-d$. Because $\phi$ preserves Euler measure, the domain $C(d, \gamma)+C(\gamma, \phi \cdot \gamma)-\phi \cdot C(d, \gamma)$ has Euler measure $m(C(\gamma, \phi \cdot \gamma))$. In addition, its boundary is $\phi \cdot d-d$. Therefore, we have a domain $C(d, \phi \cdot d)$ with boundary $\phi \cdot d-d$. Applying (6.1) with $d$ in place of $\gamma$ gives

$$
f(\phi \cdot d)-f(d)=-\frac{1}{2} m(C(d, \phi \cdot d)) \quad(\bmod g-1),
$$

while $m(C(d, \phi \cdot d))=m(C(\gamma, \phi \cdot \gamma))$ by construction. Therefore, we obtain the desired identity, and $\phi$ acts as claimed on color classes.

By Lemma 6.1, we may record the homomorphism induced by the color permutation by the value $\chi_{v}(\phi)=\frac{1}{2} m(C(d, \phi \cdot d)) \in \mathbb{Z} /(g-1) \mathbb{Z}$, where $[d]=v$. We thereby obtain a map $\chi(\phi): v \mapsto \chi_{v}(\phi)$ defined on the primitive nonzero classes $v \in H$.

Lemma 6.2. The map $\chi(\phi)$ extends to a homomorphism $\chi(\phi): H \rightarrow \mathbb{Z} /$ $(g-1) \mathbb{Z}$.

Proof. The material of Section 5.2 concerning domains and their Euler measures readily generalizes from oriented simple closed curves to multicurves, which need not be simple. In particular, for a multicurve $\gamma$ representing a 1-cycle in $Z_{1}(S ; \mathbb{Z})$ and an element $\phi \in \mathcal{I}$, there exists a domain $C$ such that $\partial C=\gamma-\phi \cdot \gamma$. It is well defined up to adding a multiple of $[S]$, so we obtain a well-defined value $\chi(\phi)(\gamma)=\frac{1}{2} m(C)(\bmod g-1)$ as before. It is clear that $\chi(\phi)$ is linear on 1 -cycles, so it defines a homomorphism $Z_{1}(S ; \mathbb{Z}) \rightarrow \mathbb{Z} /(g-1) \mathbb{Z}$. If $\gamma$ is nullhomologous, then there exists a domain $D$ such that $\partial D=\gamma$, so $D-\phi \cdot D$ is a domain with boundary $\gamma-\phi \cdot \gamma$, and we obtain

$$
\chi(\phi)(\gamma)=m(D-\phi \cdot D)=m(D)-m(\phi \cdot D)=0 .
$$


It follows that $\chi(\phi)$ descends to a homomorphism $H \rightarrow \mathbb{Z} /(g-1) \mathbb{Z}$ that extends its definition on primitive classes, as desired.

Note that $\chi(\phi)$ depends only on the mapping class of $\phi$ and that $\chi(\phi \circ \psi)=$ $\chi(\phi)+\chi(\psi)$. Thus, we obtain a homomorphism $\chi: \mathcal{I} \rightarrow \operatorname{Hom}(H, \mathbb{Z} /(g-1) \mathbb{Z})$ that records the simultaneous permutation of the $(g-1)$-colorings of all of the graphs $\mathcal{C}_{v}(S)$. Since $\chi$ has abelian image, the knowledgeable reader may rightly suspect that it factors through the Johnson homomorphism $\tau$ for $g \geqslant 3$. Indeed, the kernel of $\tau$ is generated by Dehn twists about separating curves for $g \geqslant 3$ [Joh85], so it suffices to check that $\chi$ vanishes on these. Given a Dehn twist $\phi$ about a separating curve $c$, choose a geometric symplectic basis for $S$ disjoint from $c$ (see Lemma 5.4 for a reminder of the meaning of this term). Lemmas 6.1 and 6.2 demonstrate that $\chi(\phi)$ is trivial by checking its action on this basis, certifying that $\chi$ factors through $\tau$. In the next subsection, we identify $\chi$ with a precursor to $\tau$ (Theorem 1.6). We could conclude this result at once on the basis of Remark 5.8 and Irmer's work on the relationship of signed stable length with $\tau$ [Irm15], but we include more detail for completeness.

\subsection{The coloring permutation and the Chillingworth homomorphism.} Chillingworth introduced the winding number of a regular closed curve around a nonvanishing vector field and applied its study to $\operatorname{Mod}(S)$ [Chi72a, Chi72b]. Johnson broadened and clarified Chillingworth's work, reworking the former's construction into the Chillingworth homomorphism $t$ and showing that it specializes the Johnson homomorphism $\tau$ [Joh80]. In the case of a closed surface $S$, these maps take the forms

$$
t: \mathcal{I} \rightarrow \operatorname{Hom}(H, \mathbb{Z} /(g-1) \mathbb{Z}) \quad \text { and } \quad \tau: \mathcal{I} \rightarrow \operatorname{Hom}\left(H, \wedge^{2} H\right) / j(H),
$$

where $j$ denotes a particular inclusion map. Basic properties of $j$ and the algebraic intersection pairing $\iota$ on $H$ produce a map

$$
\bar{\imath}: \operatorname{Hom}\left(H, \wedge^{2} H\right) / j(H) \rightarrow \operatorname{Hom}(H, \mathbb{Z} /(g-1) \mathbb{Z}) .
$$

The Chillingworth and Johnson homomorphisms for a closed surface are then related by the composition $t=\bar{\imath} \circ \tau$ [Joh80, Theorem 2]. Johnson also computed the image under $t$ of the bounding pair maps [Joh80, Sections $5 \& 6$ ], which generate $\mathcal{I}$ for $g \geqslant 3$. A bounding pair consists of a pair of disjoint, homologous curves $\alpha$ and $\beta$, and a bounding pair map $\phi_{\alpha, \beta}$ is the composition of a Dehn twist about $\beta$ and the inverse of a Dehn twist about $\alpha$. Johnson's result reads: 


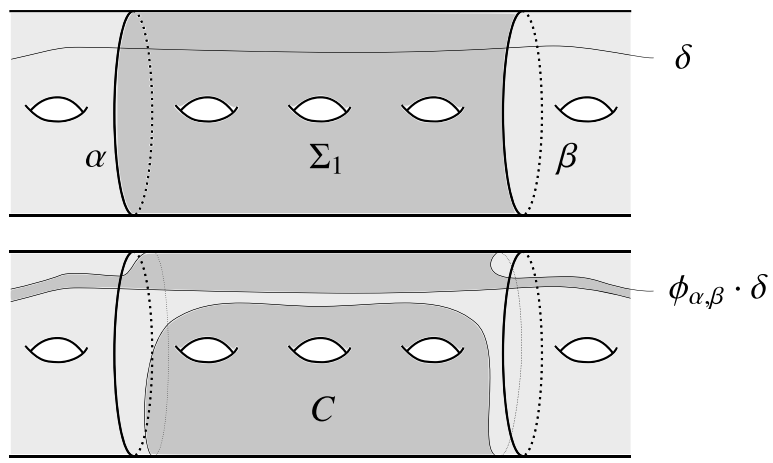

Figure 7. The bounding pair $\alpha, \beta$ cuts off the subsurface $\Sigma_{1}$, the domain $C$ has boundary $\phi_{\alpha, \beta} \cdot \delta-\delta$, and the curve $\delta$ satisfies $\chi_{[\delta]}\left(\phi_{\alpha, \beta}\right) \equiv \operatorname{genus}\left(\Sigma_{1}\right)(\bmod g-1)$.

LEMMA 6.3. Suppose that $\alpha$ and $\beta$ form a bounding pair and $S \backslash(\alpha \cup \beta)$ consists of components $\Sigma_{1}$ and $\Sigma_{2}$. The image under $t$ of the bounding pair map $\phi_{\alpha, \beta}$ is given by $t\left(\phi_{\alpha, \beta}\right)(v)=\operatorname{genus}\left(\Sigma_{1}\right) \cdot \iota(v,[\alpha])(\bmod g-1)$.

Recall Theorem 1.6:

THEOREM 1.6. The color permutation homomorphism $\chi$ equals the Chillingworth homomorphism $t$.

Proof. We follow Irmer's line of argument [Irm15, Theorem 1]. The assertion holds vacuously when $g \leqslant 2$. Since $\mathcal{I}$ is generated by bounding pair maps for $g \geqslant 3$ [Joh83], it suffices to check the assertion in this case on a bounding pair map $\phi_{\alpha, \beta}$. Choose a geometric symplectic basis for $S$ consisting of $\alpha$, a curve $\delta$ meeting $\alpha$ and $\beta$ both once apiece, and all other curves disjoint from both $\alpha$ and $\beta$. The fact that $\chi\left(\phi_{\alpha, \beta}\right)=t\left(\phi_{\alpha, \beta}\right)$ is immediate from Lemmas 6.1, 6.2, and 6.3. Figure 7 displays the single nontrivial case.

As a corollary, we can recast the coloring of $\mathcal{C}_{v}(S)$ in terms of the Torelli group and the Chillingworth homomorphism. Fix $c \in \mathcal{C}_{v}(S)$ and choose any $d \in \mathcal{C}_{v}(S)$. Because $\mathcal{I}$ acts transitively on $\mathcal{C}_{v}(S)$, there exists some $\phi_{d} \in \mathcal{I}$ so that $\phi_{d} \cdot c=d$.

COROLlaRy 6.4. The map $d \mapsto t\left(\phi_{d}\right)(v)$ coincides with the $(g-1)$-coloring $f: \mathcal{C}_{v}\left(S_{g}\right) \rightarrow \mathbb{Z} /(g-1) \mathbb{Z}$. 


\section{Arcs on planar surfaces}

In this section, we obtain analogues of our results on curve graphs for arc graphs. We specialize to the case of a planar surface $\Sigma=\Sigma_{n}$.

Let $v \in H_{1}(\Sigma, \partial \Sigma ; \mathbb{Z} / 2 \mathbb{Z})$ denote a nonzero relative homology class, and let $\mathcal{A}_{v}(\Sigma)$ denote the subgraph of $\mathcal{A}(\Sigma)$ induced on arcs that represent $v$. Note that $v$ is determined by the pair of (distinct) components of $\partial \Sigma$ containing the endpoints of any arc that represents it. Let $\mathcal{A N}(\Sigma)$ consist of the nonseparating arcs. Every arc in $\mathcal{A N}(\Sigma)$ belongs to one of the $\left(\begin{array}{l}n \\ 2\end{array}\right)$ isomorphic subgraphs $\mathcal{A}_{v}(\Sigma)$. Similarly, for a hole $\partial$ in $\Sigma$, let $\mathcal{A}_{\partial}(\Sigma)$ denote the subgraph of $\mathcal{A}(\Sigma)$ induced on arcs with both endpoints on $\partial$. Let $\mathcal{A}_{\text {sep }}(\Sigma)$ consist of the separating arcs. Every arc in $\mathcal{A}_{\text {sep }}(\Sigma)$ belongs to one of the $n$ isomorphic subgraphs $\mathcal{A}_{\partial}(\Sigma)$.

By analogy to Lemma 3.2 and with a slight adjustment to its proof, we have:

LEMmA 7.1. There exists a homomorphism $f: \mathcal{A}_{\text {sep }}\left(\Sigma_{n}\right) \rightarrow \mathrm{KG}(n)$.

Proof. Given an arc $a \in \mathcal{A}_{\text {sep }}\left(\Sigma_{n}\right)$, let $\partial$ denote the hole that contains its endpoints. The surface $\Sigma_{n} \backslash a$ consists of two components, each of which contains at least one hole of $\Sigma_{n}$. We obtain an induced partition of $\partial \Sigma_{n} \backslash \partial$ into two nontrivial parts, and we let $f(a)$ be the partition of $\partial \Sigma_{n}$ obtained by adding $\partial$ to the larger part, breaking ties arbitrarily. If $(a, b) \in \mathcal{A}_{\text {sep }}\left(\Sigma_{n}\right)$, then there are two cases to check that $f(a, b) \in \mathrm{KG}(n)$, depending on whether $a$ and $b$ have endpoints on the same hole or not. We leave the routine verification to the reader. It follows that $f$ defines the desired homomorphism.

Similarly, by analogy to Lemma 3.3, we have:

LEMMA 7.2. There exist embeddings $a_{0}: \mathrm{CG}(n-1) \hookrightarrow \mathcal{A}_{\partial}\left(\Sigma_{n}\right)$ and $a_{1}$ : $\mathrm{CG}(n) \backslash \mathrm{CG}(n, 1) \hookrightarrow \mathcal{A N}\left(\Sigma_{n}\right)$.

Proof. For the first part, realize $\Sigma_{n}$ as a round disk $D$ with $n-1$ evenly spaced small holes $\partial_{1}, \ldots, \partial_{n-1}$ near $\partial D$. For $1 \leqslant i<j \leqslant n-1$, let $a_{0}(i, j)$ denote a chord of $D$ disjoint from these holes and that partitions them into two parts, one of which consists of $\partial_{i}, \ldots, \partial_{j-1}$. See Figure 8 . Note that the isotopy type of $a_{0}(i, j)$ is well defined. Furthermore, $a_{0}(i, j)$ and $a_{0}\left(i^{\prime}, j^{\prime}\right)$ can be made disjoint if and only if $(i, j)$ and $\left(i^{\prime}, j^{\prime}\right)$ are unlinked. It follows that $a_{0}$ defines the desired embedding.

For the second part, realize $\Sigma_{n}$ as a sphere $\mathbb{R}^{2} \cup\{\infty\}$ with $n$ evenly spaced small holes $\partial_{1}, \ldots, \partial_{n}$ around a circle. For $1 \leqslant i<j \leqslant n$, let $a_{1}(i, j)$ denote a line segment with endpoints on $\partial_{i}$ and $\partial_{j}$. As in the first part, it follows that $a_{1}$ defines the desired embedding. 


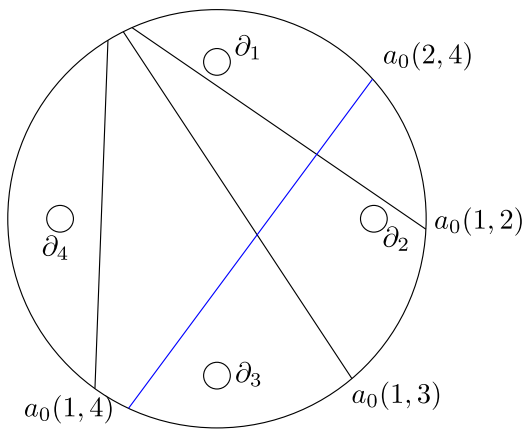

Figure 8 . Four arcs in the image of $a_{0}$. The pairs $(1, j)$ and $(2,4)$ are linked for $j \in\{2,3\}$.

THEOREM 7.3. $\chi\left(\mathcal{A}_{\text {sep }}\left(\Sigma_{n}\right)\right) \sim n \log n$.

Proof. The result follows from the sequence of homomorphisms

$$
\mathrm{CG}(n-1) \stackrel{a_{0}}{\hookrightarrow} A_{\partial}\left(\Sigma_{n}\right) \hookrightarrow \mathcal{A}_{\mathrm{sep}}\left(\Sigma_{n}\right) \stackrel{f}{\rightarrow} \mathrm{KG}(n)
$$

provided by Lemmas 7.1 and 7.2, as well as one more application of Theorem 1.1 and the monotonicity of chromatic numbers.

Doubling the surface $\Sigma_{n}$ along its boundary results in a closed surface $S_{n-1}$. Every arc $a \in \mathcal{A}\left(\Sigma_{n}\right)$ gets doubled to a curve $d(a) \in \mathcal{C}\left(S_{n-1}\right)$. If $a^{\prime}$ is another arc that represents the same $(\bmod 2)$ relative homology class as $a$, then $a$ and $a^{\prime}$ can be simultaneously oriented so that they represent the same integral relative homology class. A 2-chain with boundary $a-a^{\prime}$ doubles to a 2-chain with boundary $d(a)-d\left(a^{\prime}\right)$, where these curves inherit orientations from the arcs. It follows that a $(\bmod 2)$ relative class $v$ represented by an arc $a$ doubles to a nonzero class $w \in H_{1}\left(S_{n-1} ; \mathbb{Z}\right)$, which is well defined up to sign. The map $d: \mathcal{A}\left(\Sigma_{n}\right) \rightarrow \mathcal{C}\left(S_{n-1}\right)$ defines an embedding, and it restricts to an embedding $\mathcal{A}_{v}\left(\Sigma_{n}\right) \hookrightarrow \mathcal{C}_{w}\left(S_{n-1}\right)$.

THEOREM 7.4. $\chi\left(\mathcal{A}_{v}\left(\Sigma_{n}\right)\right)=\omega\left(\mathcal{A}_{v}\left(\Sigma_{n}\right)\right)=n-2$, and $\mathcal{A}_{v}\left(\Sigma_{n}\right)$ is uniquely $(n-2)$-colorable.

Proof. The embedding $\mathcal{A}_{v}\left(\Sigma_{n}\right) \hookrightarrow \mathcal{C}_{w}\left(S_{n-1}\right)$ and Theorem 5.12 show that $\mathcal{A}_{v}\left(\Sigma_{n}\right)$ is $(n-2)$-colorable. The proof of uniqueness follows the paradigm of Section 5.4 once more. We realize $\Sigma_{n}$ as an annulus with $n-2$ equally spaced 


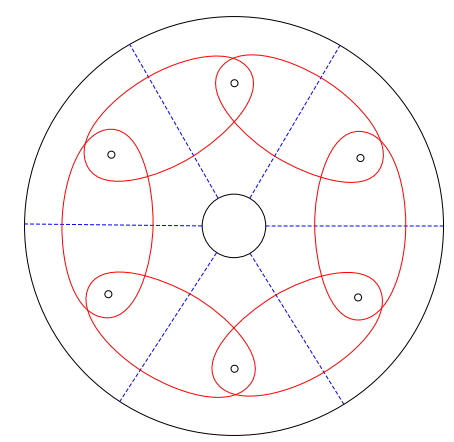

(a) The dashed blue arcs form a maximal clique $c_{0} \in \mathcal{K}\left(\mathcal{A}_{v}\left(\Sigma_{n}\right)\right)$.

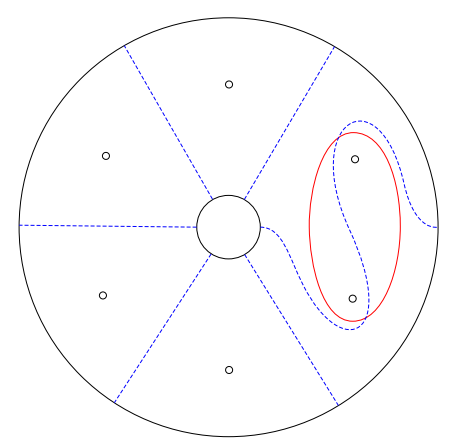

(b) The action of a half Dehn twist on $c_{0}$.

Figure 9. Half Dehn twists about the red curves on the left together with a rotation by angle $2 \pi /(n-2)$ generate the annular braid group.

small holes. Let $\partial_{i}$ and $\partial_{j}$ denote the boundary components of the annulus and let $v$ denote the class of arc with one endpoint on each of $\partial_{i}$ and $\partial_{j}$. The subgroup $P_{i j}<\operatorname{Mod}\left(\Sigma_{n}\right)$ that fixes each of $\partial_{i}$ and $\partial_{j}$ setwise is the annular braid group on $n-2$ strands. It is generated by the $n-2$ swaps of consecutive holes by half Dehn twists and the homeomorphism of $\Sigma_{n}$ induced by rotation of the annulus through the angle $2 \pi /(n-2)$ [KP02, Theorem 1]. Choosing the clique $c_{0} \in \mathcal{K}\left(\mathcal{A}_{v}\left(\Sigma_{n}\right)\right)$ given by $n-2$ radial segments, we see that the image of $c_{0}$ under each generator lies in the same connected component of $\mathcal{K}\left(\mathcal{A}_{v}\left(\Sigma_{n}\right)\right)$ as $c_{0}$. See Figure 9. As $P_{i j}$ acts transitively on vertices of $\mathcal{K}\left(\mathcal{A}_{v}\left(\Sigma_{n}\right)\right)$, Putman's technique shows that $\mathcal{K}\left(\mathcal{A}_{v}\left(\Sigma_{n}\right)\right)$ is connected. Finally, the complex $\mathcal{A}_{v}\left(\Sigma_{n}\right)$ is pure of dimension $n-3$, which also implies that $\omega\left(\Sigma_{n}\right)=n-2$. Lemma 5.14 closes the proof.

Recall Theorem 1.7:

THEOREM 1.7. $n \log n \lesssim \chi\left(\mathcal{A}\left(\Sigma_{n}\right)\right) \lesssim n^{3}$.

Proof of Theorem 1.7. The arc graph $\mathcal{A}\left(\Sigma_{n}\right)$ is the vertex-disjoint union of $\mathcal{A}_{\text {sep }}\left(\Sigma_{n}\right)$ and the $\left(\begin{array}{l}n \\ 2\end{array}\right)$ subgraphs $\mathcal{A}_{v}\left(\Sigma_{n}\right)$. The result now follows from the union bound and Theorems 7.3 and 7.4.

REMARK 7.5. The order of growth of the upper bound in Theorem 1.7 is dominated by the nonseparating arcs, so it is tempting to explore more judicious colorings of nonseparating arcs of different types. Proposition 8.3 and Theorem 8.6 in the next Section take first steps in this direction. 


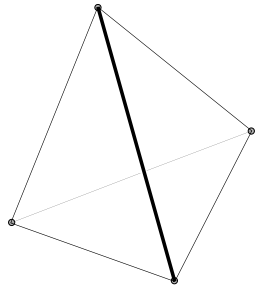

(a) An arc in $V_{1}$.

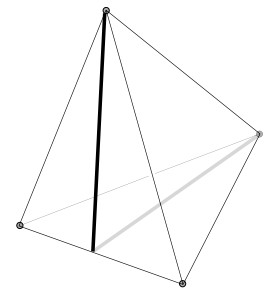

(b) An arc in $V_{2}$.

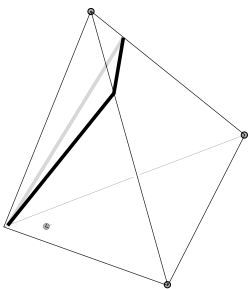

(c) An arc in $V_{3}$.

Figure 10. A schematic of the $\operatorname{arcs} V \subset \mathcal{A}\left(\Sigma_{4}\right)$.

\section{The four-holed sphere}

In this section, we study the arc graph of the four-holed sphere $\Sigma_{4}$ and obtain some exact results. Although we cannot pin down the exact value of $\chi\left(\mathcal{A}\left(\Sigma_{4}\right)\right)$ (Theorem 8.4), we determine the chromatic number of the subgraphs of $\mathcal{A}\left(\Sigma_{4}\right)$ induced on separating arcs (Proposition 8.2), nonseparating arcs (Proposition 8.3), and nonseparating arcs with one endpoint on a fixed hole (Theorem 8.6).

LEMMA 8.1. $\chi_{f}\left(\mathcal{A}\left(\Sigma_{4}\right)\right) \geqslant 22 / 3$.

Proof. Identify $\Sigma_{4}$ with the 2-skeleton of a regular tetrahedron in $\mathbb{R}^{3}$ with holes placed at its vertices. Let $V_{1}$ denote the arcs of $\Sigma_{4}$ determined by the edges of the tetrahedron. There are six reflections of $\Sigma_{4}$, and each has a fixed point set consisting of two arcs of $\Sigma_{4}$. From this pair, one arc is in $V_{1}$; let $V_{2}$ consist of the six other arcs of $\Sigma_{4}$ determined by these six reflections. Let $V_{3}$ consist of the twelve (isotopy classes of) arcs of $\Sigma_{4}$ determined by the intersection of $\Sigma_{4}$ with a plane passing through exactly one vertex of the tetrahedron. Finally, consider the subgraph $G$ induced on the vertex set $V=V_{1} \cup V_{2} \cup V_{3}$. See Figure 10.

Let $I$ be a maximal independent set of $G$, and let $a_{i}=\left|I \cap V_{i}\right|$ for $i=1,2$, 3. It is straightforward to check that $\left(a_{1}, a_{2}, a_{3}\right) \in\{(1,1,0),(1,0,2),(0,1,2)$, $(0,1,4),(0,2,2),(0,2,3),(0,3,1),(0,3,3)\}$. A function $f: V \rightarrow \mathbb{R}_{\geqslant 0}$ satisfying $\left.f\right|_{V_{i}}=c_{i}$, for $i=1,2,3$, is a fractional clique if $\left(c_{1}, c_{2}, c_{3}\right) \cdot\left(a_{1}, a_{2}\right.$, $\left.a_{3}\right) \leqslant 1$ for all $\left(a_{1}, a_{2}, a_{3}\right)$ as above. Thus, taking $c_{1}=7 / 9, c_{2}=2 / 9, c_{3}=1 / 9$ yields a fractional clique of total value $\sum c_{i}\left|V_{i}\right|=6 c_{1}+6 c_{2}+12 c_{3}=22 / 3$, and the lemma follows.

Proposition 8.2. $\chi\left(\mathcal{A}_{\text {sep }}\left(\Sigma_{4}\right)\right)=\omega\left(\mathcal{A}_{\text {sep }}\left(\Sigma_{4}\right)\right)=3$. 
Proof. Mark one of the holes. One of the components in the complement of a separating arc $a$ contains a single hole of $\Sigma_{4}$. If it is not marked, let $c(a)$ denote this hole; if it is, let $c(a)$ denote the hole containing the endpoints of $a$. This function $c$ defines a proper 3-coloring, and it is easy to locate a clique of size 3.

Proposition 8.3. $\chi\left(\mathcal{A N}\left(\Sigma_{4}\right)\right)=\omega\left(\mathcal{A N}\left(\Sigma_{4}\right)\right)=6$.

Proof. Write $\partial \Sigma_{4}=\partial_{1} \cup \partial_{2} \cup \partial_{3} \cup \partial_{4}$. Let $\mathcal{A}_{i j}\left(\Sigma_{4}\right)$ denote the subgraph induced on arcs with one endpoint on $\partial_{i}$ and the other on $\partial_{j}$. Note that an arc in $\mathcal{A}_{12}\left(\Sigma_{4}\right)$ is disjoint from a unique arc in $\mathcal{A}_{34}\left(\Sigma_{4}\right)$. Fix such a pair $\left(a_{12}, a_{34}\right)$. Using Theorem 7.4, 2-color both of $\mathcal{A}_{12}\left(\Sigma_{4}\right)$ and $\mathcal{A}_{34}\left(\Sigma_{4}\right)$ so that $a_{12}$ and $a_{34}$ get opposite colors. One checks that any arc in $\mathcal{A}_{12}\left(\Sigma_{4}\right)$ gets the opposite color from the unique arc that it is disjoint from in $\mathcal{A}_{34}\left(\Sigma_{4}\right)$. Thus, we obtain a proper 2-coloring of the subgraph induced on $\mathcal{A}_{12}\left(\Sigma_{4}\right) \cup \mathcal{A}_{34}\left(\Sigma_{4}\right)$. Copy this coloring onto the other two subgraphs corresponding to the partitions of the holes into two pairs, using a different palette for each. The result is the desired 6-coloring. The arcs in $V_{1}$ described in the proof of Lemma 8.1 form a clique of size 6.

THEOREM 8.4. $8 \leqslant \chi\left(\mathcal{A}\left(\Sigma_{4}\right)\right) \leqslant 9$.

Proof. Immediate from Lemma 8.1 and Propositions 8.2 and 8.3.

As a first step toward improving the upper bound in Theorem 1.7, it is possible to color a subcomplex of the nonseparating arcs with fewer colors than those used above. Let $\mathcal{A}^{\prime} \subset \mathcal{A}\left(\Sigma_{4}\right)$ denote the subcomplex induced on arcs with exactly one endpoint on a fixed hole $\partial_{i}$, and note that six colors are used to color $\mathcal{A}^{\prime}$ in the coloring of Proposition 8.3. We demonstrate in Theorem 8.6 that four suffice.

We begin by relating $\mathcal{A}^{\prime}$ to $\mathcal{C}\left(\Sigma_{4}\right)$. By definition, a simplex in $\mathcal{C}\left(\Sigma_{4}\right)$ consists of curves with pairwise minimal intersection number 2 . There exists a well-known isomorphism between the complex $\mathcal{C}\left(\Sigma_{4}\right)$ and the Farey complex $\mathcal{F}$ [FM11, pp. 94-95]. The vertex set of $\mathcal{F}$ is $P^{1}\left(\mathbb{Z}^{2}\right)$, the set of lines in $\mathbb{Z}^{2}$. Given a pair of lines $L_{1}, L_{2} \in P^{1}\left(\mathbb{Z}^{2}\right)$, let $d\left(L_{1}, L_{2}\right)$ denote the index in $\mathbb{Z}^{2}$ of the subgroup generated by their elements. The edge set of $\mathcal{F}$ consists of all pairs $\left(L_{1}, L_{2}\right)$ satisfying $d\left(L_{1}, L_{2}\right)=1$. It is a flag complex, and it is pure of dimension 2 . The group PSL $(2, \mathbb{Z})$ acts on $P^{1}\left(\mathbb{Z}^{2}\right)$ and by extension on $\mathcal{F}$.

Form the pure, 3-dimensional supercomplex $\mathcal{F}^{\prime} \supset \mathcal{F}$ whose 3-simplices are the unions of the 2-simplices in $\mathcal{F}$ that share a common edge. Given $\left(L_{1}, L_{2}\right) \in E(\mathcal{F})$, with $L_{i}$ generated by $x_{i} \in \mathbb{Z}^{2}$, there exist two maximal simplices in $\mathcal{F}$ containing $\left(L_{1}, L_{2}\right)$, and they take the form $\left(L_{1}, L_{2}, L^{ \pm}\right)$, where $L^{ \pm}$is generated by $x_{1} \pm x_{2}$. 
It follows that $d\left(L^{+}, L^{-}\right)=2$. Thus, for every edge $\left(L, L^{\prime}\right) \in E\left(\mathcal{F}^{\prime}\right)$, we have $d\left(L, L^{\prime}\right) \in\{1,2\}$. Observe that $\operatorname{PSL}(2, \mathbb{Z})$ extends to an action on $\mathcal{F}^{\prime}$.

Proposition 8.5. $\mathcal{A}^{\prime} \approx \mathcal{F}^{\prime}$.

Proof. An arc in $\mathcal{A}^{\prime}$ is disjoint from a unique curve in $\mathcal{C}\left(\Sigma_{4}\right)$, and vice versa. In this way, we obtain a natural bijection between the vertex sets of these complexes. Moreover, disjoint arcs in $\mathcal{A}^{\prime}$ on different endpoint pairs correspond to curves in $\mathcal{C}\left(\Sigma_{4}\right)$ with minimal geometric intersection number 2 , and vice versa. It follows that $\mathcal{F} \approx \mathcal{C}\left(\Sigma_{4}\right)$ naturally embeds as a subcomplex of $\mathcal{A}^{\prime}$. The 2-simplices of $\mathcal{A}^{\prime}$ contained in $\mathcal{F}$ are spanned by triples of pairwise disjoint arcs in $\mathcal{A}^{\prime}$ on different pairs of endpoints. Given two such 2-simplices that share a common edge, the pair of vertices in these simplices not on the shared edge are disjoint arcs in $\mathcal{A}^{\prime}$ with the same pair of endpoints. Therefore, the four vertices in the union of these 2simplices span a 3-simplex in $\mathcal{A}^{\prime}$. It follows that $\mathcal{F}^{\prime} \subset \mathcal{A}^{\prime}$. Conversely, a maximal simplex $\sigma \subset \mathcal{A}^{\prime}$ consists of four pairwise disjoint arcs, precisely two of which have the same endpoint pair. Thus, it contains two 2-simplices in $\mathcal{F}$ that meet along an edge, so $\sigma \subset \mathcal{F}^{\prime}$. It follows that $\mathcal{A}^{\prime} \subset \mathcal{F}^{\prime}$, and the proof is complete.

Recall that for a positive integer $n$, the congruence subgroup $\Gamma(n) \subset \operatorname{PSL}(2, \mathbb{Z})$ is the kernel of the natural epimorphism $\operatorname{PSL}(2, \mathbb{Z}) \rightarrow \operatorname{PSL}(2, \mathbb{Z} / n \mathbb{Z})$ obtained by reducing $(\bmod n)$.

THEOREM 8.6. $\chi\left(\mathcal{A}^{\prime}\right)=\chi\left(\mathcal{F}^{\prime}\right)=4$. Moreover, the $\Gamma$ (3)-orbits under the action by $\operatorname{PSL}(2, \mathbb{Z})$ on $P^{1}\left(\mathbb{Z}^{2}\right)$ comprise the color classes in a proper 4 -coloring of $\mathcal{F}^{\prime}$.

Proof. Since $\mathcal{F}^{\prime}$ is 3-dimensional, $\chi\left(\mathcal{F}^{\prime}\right) \geqslant 4$ follows at once. Next, map a line in $P^{1}\left(\mathbb{Z}^{2}\right)$ to its $(\bmod 3)$ reduction in $P^{1}\left((\mathbb{Z} / 3 \mathbb{Z})^{2}\right)$. Observe that $P^{1}\left((\mathbb{Z} / 3 \mathbb{Z})^{2}\right)$ consists of four lines. Given an edge $\left(L_{1}, L_{2}\right) \in E\left(\mathcal{F}^{\prime}\right), d\left(L_{1}, L_{2}\right)$ is relatively prime to 3 , so the subgroup generated by the elements of $L_{1}$ and $L_{2}$, reduced $(\bmod 3)$, is all of $(\mathbb{Z} / 3 \mathbb{Z})^{2}$. In particular, $L_{1}$ and $L_{2}$ reduce to distinct lines $(\bmod 3)$. Therefore, the $(\bmod 3)$ reduction map defines a proper 4 -coloring of $\mathcal{F}^{\prime}$, and $\chi\left(\mathcal{F}^{\prime}\right)=4$, as desired.

Next, the transitive action by $\operatorname{PSL}(2, \mathbb{Z})$ on $\mathcal{F}^{\prime}$ permutes the color classes under the 4-coloring $f$ just described. Let $g \in \operatorname{PSL}(2, \mathbb{Z})$ and select a line $L \in P^{1}\left(\mathbb{Z}^{2}\right)$. We have $f(g \cdot L)=\bar{g} \cdot f(L)$, where $\bar{g} \in P S L(2, \mathbb{Z} / 3 \mathbb{Z})$ denotes the reduction of $g$ $(\bmod 3)$. It follows that the subgroup of $\operatorname{PSL}(2, \mathbb{Z})$ that preserves the color classes consists of those group elements $g$ for which $\bar{g}$ fixes all lines in $(\mathbb{Z} / 3 \mathbb{Z})^{2}$. Such an element $\bar{g}$ is represented by a diagonal matrix in $\operatorname{SL}(2, \mathbb{Z} / 3 \mathbb{Z})$, which forces $\bar{g}= \pm I$. Therefore, the color-preserving subgroup of $\operatorname{PSL}(2, \mathbb{Z})$ is precisely $\Gamma(3)$, which completes the proof. 


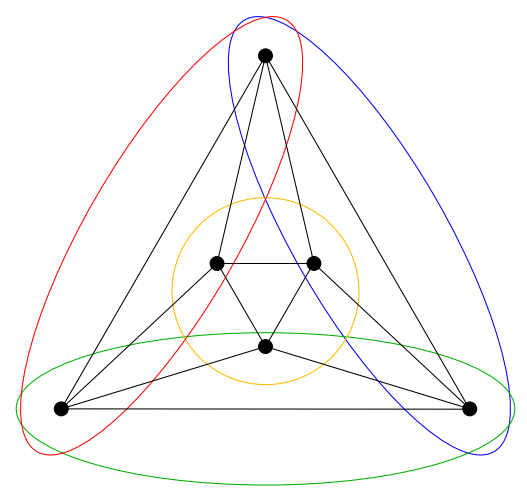

Figure 11. The octahedron and four bisecting circles, colored solely for visual aid.

In the language of this proof, the unique 3 -coloring of $\mathcal{F}$ comes by mapping each line to its $(\bmod 2)$ reduction; the color classes are the $\Gamma(2)$-orbits.

\section{The genus two surface}

In this section, we study the chromatic number of the curve graph of the closed surface of genus two and prove Theorem 1.8:

THEOREM 1.8. $\chi\left(\mathcal{N}\left(S_{2}\right)\right)=4$ and $\chi\left(\mathcal{C}\left(S_{2}\right)\right)=5$.

We first consider a pair of finite graphs that we use to obtain the required lower bounds. We then apply a homomorphism out of $\mathcal{N}\left(S_{2}\right)$ defined using hyperbolic geometry in order to obtain the required upper bounds. This homomorphism admits an alternative description in terms of homology, as we show in Proposition 9.2.

Consider the octahedron $O$ and the four circles appearing in Figure 11. The four circles separate the four pairs of antipodal faces in $O$. Let $E$ denote the set of edges of $O$ and $C$ the set of circles. Let $\mathcal{C}$ denote the graph with vertex set $E \cup C$, where adjacency connotes disjointness; edges with a common endpoint are not disjoint. Let $\mathcal{N}$ denote the subgraph of $\mathcal{C}$ induced on $E$. Thus, $\mathcal{N}$ is the complement of the line graph of $O$.

Proposition 9.1. $\chi(\mathcal{N})=4$ and $\chi(\mathcal{C})=5$.

Proof. The fact that $\chi(\mathcal{N})=4$ is well known. We obtain $\chi(\mathcal{C}) \leqslant 5$ by properly 4-coloring $\mathcal{N}$ and giving every circle a common fifth color. It is straightforward to 
check that an independent set in $\mathcal{C}$ contains at most four elements, with equality if and only if they are four edges meeting at a vertex or four circles. As $|E \cup C|=16$, if there were a 4-coloring of $\mathcal{C}$, then each color class must contain four elements. Thus, one color class must consist of four circles, so the remaining ones induce a 3 -coloring of $\mathcal{N}$, a contradiction. Thus, $\chi(\mathcal{C}) \geqslant 5$, completing the proof.

Proof of Theorem 1.8. Place a hyperbolic structure on $S_{2}$. It admits a unique hyperelliptic involution $J$ with six fixed points (see the discussions following [FM11, Theorem 3.10 \& Proposition 7.15]). Every nonseparating geodesic $\alpha \subset S_{2}$ meets $\operatorname{Fix}(J)$ in precisely two points [MRT14, Proposition 2.3]. This result follows at once from [HS89, Theorem 1], according to which $J(\alpha)=\alpha$ and $J$ reverses the orientation on $\alpha$ : it follows that $J \mid \alpha$ is a reflection, so it has two fixed points. Clearly, disjoint nonseparating geodesics must meet $\operatorname{Fix}(J)$ in distinct pairs of points. Therefore, the assignment of a nonseparating geodesic to the pair of points it meets in Fix $(J)$ defines a homomorphism $h: \mathcal{N}\left(S_{2}\right) \rightarrow \operatorname{KG}(6,2)$. Since $\chi(\operatorname{KG}(6,2))=4, h$ induces a proper 4-coloring of $\mathcal{N}\left(S_{2}\right)$. Any two curves in $\mathcal{C}_{\text {sep }}\left(S_{2}\right)$ intersect, so giving them a common fifth color leads to a proper 5coloring of $\mathcal{C}\left(S_{2}\right)$. Thus, we obtain the required upper bounds $\chi\left(\mathcal{N}\left(S_{2}\right)\right) \leqslant 4$ and $\chi\left(\mathcal{C}\left(S_{2}\right)\right) \leqslant 5$.

Next, form the double cover of the two-sphere branched along the six vertices of $O$. The result is a surface homeomorphic to $S_{2}$. The edges in $E$ lift to nonseparating curves, and the circles in $C$ lift to separating curves. The subgraph of $\mathcal{C}\left(S_{2}\right)$ induced on their lifts is isomorphic to $\mathcal{C}$, while the subgraph induced on the lifts of $E$ is isomorphic to $\mathcal{N}$, leading to the required lower bounds $\chi\left(\mathcal{N}\left(S_{2}\right)\right) \geqslant 4$ and $\chi\left(\mathcal{C}\left(S_{2}\right)\right) \geqslant 5$.

We now turn to a nongeometric description of the map $h$. The $(\bmod 2)$ intersection pairing $\widehat{\imath}$ equips $H_{1}\left(S_{g} ; \mathbb{F}_{2}\right)$ with the structure of a symplectic vector space. Let $G_{2 g}$ denote the graph with vertex set the nonzero elements of $H_{1}\left(S_{g} ; \mathbb{F}_{2}\right)$, where two distinct elements span an edge if they are orthogonal with respect to $\widehat{\iota}$. There exists a natural map $f: \mathcal{N}\left(S_{g}\right) \rightarrow G_{2 g}$ assigning a curve to its $(\bmod 2)$ homology class. This map is a relative of the natural homomorphism $\mathcal{C}\left(\Sigma_{n}\right) \rightarrow \operatorname{KG}(n)$ studied in the case of a planar surface. The map $f$ is a homomorphism when $g=2$, since distinct homologous curves intersect, but it is not for any value $g \geqslant 3$.

Proposition 9.2. The homomorphisms $f: \mathcal{N}\left(S_{2}\right) \rightarrow G_{4}$ and $h: \mathcal{N}\left(S_{2}\right) \rightarrow$ $\mathrm{KG}(6,2)$ coincide in the sense that there exists an isomorphism $\varphi: \operatorname{KG}(6,2) \rightarrow$ $G_{4}$ such that $\varphi \circ h=f$. In particular, up to automorphism of $\operatorname{KG}(6,2), h$ does not depend on the choice of hyperbolic structure on $S_{2}$. 
The existence of an isomorphism $G_{4} \approx \operatorname{KG}(6,2)$ follows from the fact that both are strongly regular graphs with parameters $(15,6,1,3)$, of which there exists a unique isomorphism type [CvL91, Theorems (2.3) and (4.14)], [vLW01, remark after Theorem 21.5]. Stripping the proof of Proposition 9.2 to its algebraic core establishes this isomorphism directly. The fact that $h$ does not depend on the choice of hyperbolic structure follows as well from the connectivity of Teichmüller space.

For context, we begin in somewhat greater generality. Fix $g \geqslant 0$ and let $P=$ $\left\{p_{1}, \ldots, p_{2(g+1)}\right\}$ denote a set of marked points on a sphere $S$. Define a bilinear pairing $b$ on $H_{0}\left(P ; \mathbb{F}_{2}\right)$ by the rule $b\left(\left[p_{i}\right],\left[p_{j}\right]\right)=\delta_{i j}$. The pairing $b$ restricts to $[P]^{\perp}$, on which its annihilator is generated by $[P]=\sum_{i}\left[p_{i}\right]$. Thus, $b$ descends to a nondegenerate bilinear pairing $\bar{b}$ on $[P]^{\perp} /([P])$, a $(2 g)$-dimensional vector space.

LEMMA 9.3. There exists an isomorphism $\left([P]^{\perp} /([P]), \bar{b}\right) \approx\left(H_{1}\left(\Sigma(S, P) ; \mathbb{F}_{2}\right)\right.$, $\widehat{\imath})$, where $\Sigma(S, P) \approx S_{g}$ denotes the double cover of $S$ branched along $P$.

Proof. We will construct the required isomorphism as the composition of a boundary map and a transfer map.

The inclusion-induced map $H_{0}\left(P ; \mathbb{F}_{2}\right) \rightarrow H_{0}\left(S ; \mathbb{F}_{2}\right)$ has kernel $[P]^{\perp}$. Thus, the long exact sequence in homology of the pair $(S, P)$ leads to the identification $\partial_{*}: H_{1}\left(S, P ; \mathbb{F}_{2}\right) \stackrel{\sim}{\rightarrow}[P]^{\perp}$. We use the identification by $\partial_{*}$ to endow $H_{1}\left(S, P ; \mathbb{F}_{2}\right)$ with a bilinear pairing $b^{\prime}$. Thus, $\partial_{*}$ determines an isomorphism of inner product spaces

$$
\partial_{*}:\left(H_{1}\left(S, P ; \mathbb{F}_{2}\right), b^{\prime}\right) \stackrel{\sim}{\rightarrow}\left([P]^{\perp}, b\right) .
$$

On the level of relative 1-cycles, $b^{\prime}$ takes a pair of relative 1-cycles and returns the (mod 2) count of the number of points in which their boundaries meet.

There is a transfer map on chains $C_{1}\left(S, P ; \mathbb{F}_{2}\right) \rightarrow C_{1}\left(\Sigma(S, P) ; \mathbb{F}_{2}\right)$ obtained by mapping a relative 1-chain on $(S, P)$ to the sum of its two lifts to $\Sigma(S, P)$. The transfer map on chains descends to a transfer homomorphism on homology $\tau: H_{1}\left(S, P ; \mathbb{F}_{2}\right) \rightarrow H_{1}\left(\Sigma(S, P) ; \mathbb{F}_{2}\right)$. Observe that a pair of transverse relative 1cycles on $(S, P)$ whose boundaries meet in $k$ points of $P$ lifts to a pair of 1-cycles on $\Sigma(S, P)$ that meet in $k(\bmod 2)$ transverse points of intersection. Therefore, $\tau$ determines a map of inner product spaces

$$
\tau:\left(H_{1}\left(S, P ; \mathbb{F}_{2}\right), b^{\prime}\right) \rightarrow\left(H_{1}\left(\Sigma(S, P) ; \mathbb{F}_{2}\right), \widehat{\iota}\right) .
$$

We claim that the map

$$
\tau \circ\left(\partial_{*}\right)^{-1}:\left([P]^{\perp}, b\right) \rightarrow\left(H_{1}\left(\Sigma(S, P) ; \mathbb{F}_{2}\right), \widehat{\iota}\right)
$$


surjects with kernel generated by $[P]$, which gives the desired result. Choose a collection of $\operatorname{arcs} \gamma_{1}, \ldots, \gamma_{2(g+1)} \subset(S, P)$ such that $\partial_{*}\left[\gamma_{i}\right]=\left[p_{i}\right]+\left[p_{i+1}\right]$ for all $i$, indices $(\bmod 2(g+1))$, and for which any pair meets solely in their endpoints. Their classes form a basis for $H_{1}\left(S, P ; \mathbb{F}_{2}\right)$ subject to the single relation $\sum_{i}\left[\gamma_{i}\right]=0$. The classes $\tau\left[\gamma_{1}\right], \ldots, \tau\left[\gamma_{2(g+1)}\right]$ span $H_{1}\left(\Sigma(S, P) ; \mathbb{F}_{2}\right)$, and they obey the two relations $\sum_{i} \tau\left[\gamma_{i}\right]=0$ and $\sum_{i \equiv 0(\bmod 2)} \tau\left[\gamma_{i}\right]=0$. Thus, $\tau$ surjects and has kernel generated by $\sum_{i \equiv 0(\bmod 2)}\left[\gamma_{i}\right]$. Since $\partial_{*} \sum_{i \equiv 0(\bmod 2)}\left[\gamma_{i}\right]=[P]$, the stated property of $\tau \circ\left(\partial_{*}\right)^{-1}$ now follows and completes the proof.

Proof of Proposition 9.2. Apply Lemma 9.3 to the case $g=2$. Each nonzero class in $[P]^{\perp} /([P])$ is uniquely represented by an element of the form $\left[p_{i}\right]+\left[p_{j}\right]$ for distinct $i, j \in\{1, \ldots, 6\}$, and two such elements are $\bar{b}$-orthogonal if and only if they are equal or correspond to disjoint 2-element subsets. Let $\varphi: \operatorname{KG}(6,2) \rightarrow G_{4}$ denote the map assigning the subset $\{i, j\}$ to $\tau\left(\left[p_{i}\right]+\left[p_{j}\right]\right)$. Lemma 9.3 shows that $\varphi$ is an isomorphism.

Next, select a nonseparating, simple, closed geodesic $\alpha \subset S_{2}$. It meets $\operatorname{Fix}(J)$ in two points. Let $P$ denote the points covered by $\operatorname{Fix}(J)$. The image of $\alpha$ in the quotient $S_{2} / J$ is a simple arc $a$ that meets $P$ precisely in its endpoints $p_{i}$ and $p_{j}$. Thus, $h(\alpha)=\{i, j\}$, and $\varphi(h(\alpha))=[\alpha]=f(\alpha)$. Therefore, $\varphi \circ h=f$.

\section{Problems for further study}

Here we collect some problems of varying difficulty for further study. The first one is the most prominent:

PROBLEM 10.1. Improve the estimates on the (fractional) chromatic numbers of $\mathcal{C}\left(S_{g}\right)$ and $\mathcal{A}\left(\Sigma_{n}\right)$ in Theorems 1.5 and 1.7. We believe that both are closer to the stated lower bounds.

Problem 10.2. For $n>2 k$, let $\mathcal{C}(n, k) \leqslant \mathcal{C}\left(\Sigma_{n}\right)$ denote the subgraph induced on the curves that cut off exactly $k$ holes to one side. The natural homomorphism $\mathcal{C}(n, k) \rightarrow \operatorname{KG}(n, k)$ leads to the bound $\chi(\mathcal{C}(n, k)) \leqslant n-2 k+2$. Does equality hold? This is an analogue to Kneser's conjecture for curve graphs. We do not expect $\mathcal{C}(n, k)$ to contain a subgraph isomorphic to $\mathrm{KG}(n, k)$ (compare Proposition 3.4).

Problem 10.3. Determine the exact value of $\chi(\mathrm{KG}(n))$. Does it equal the upper bound given in Theorem 2.5?

Problem 10.4. What is $\chi\left(\mathcal{A}\left(\Sigma_{4}\right)\right)$ ? It is 8 or 9 by Theorem 8.4 . 
Problem 10.5. Generalize the results on unique colorability. For instance, does there exist a unique homomorphism $\mathcal{C}\left(\Sigma_{n}\right) \rightarrow \mathrm{KG}(n) \backslash \mathrm{KG}(n, 1)$ ? Compare this question with the rigidity of embeddings $\mathrm{CG}(n) \backslash \mathrm{CG}(n, 1) \hookrightarrow \mathcal{C}\left(\Sigma_{n}\right)$ [AL13]. Does there exist a unique homomorphism $\mathcal{N}\left(S_{2}\right) \rightarrow \mathrm{KG}(6,2)$ ? Proposition 9.2 provides evidence for this possibility.

PROBLEM 10.6. Explore other graph-theoretic properties of the curve graphs related to the chromatic number, such as the Shannon capacity and spectra.

Problem 10.7. Identify $\operatorname{Aut}\left(\mathcal{C}_{v}(S)\right.$ ) with a subgroup of $\operatorname{Mod}(S)$ (see [Iva97]). Is it isomorphic to the stabilizer of $v$ in $\operatorname{Mod}(S)$ ? Does there exist a simple generating set for this stabilizer analogous to the Humphries generating set? In that way, it might be possible to recover the closed case of Proposition 5.13 without passage to the case of nonempty boundary.

PROBLEM 10.8. Describe a generating set for the kernel of the Chillingworth homomorphism. Is it generated by the Johnson kernel and $(g-1)$ th powers of bounding pair maps? (It is finitely generated because it is a finite-index subgroup of the Torelli group, itself finitely generated by [Joh83].)

\section{Acknowledgements}

We thank Ian Biringer and Peter Feller for pleasant conversations throughout the course this work and Ken Bromberg for helpful email correspondence. We especially thank Ian for explaining how to use train tracks in place of hyperbolic geometry in the proof of Theorem 1.3. We also thank the referees for their thorough reviews. One spotted a lemma that was simply incorrect, which we subsequently removed and bypassed. NGV is grateful to Peter Heinig for bringing the chromatic number of the curve graph to his attention. JEG was supported by NSF CAREER Award DMS-1455132 and an Alfred P. Sloan Foundation Research Fellowship. NGV was supported in part by NSF RTG grant 1045119.

\section{References}

[ABG17] T. Aougab, I. Biringer and J. Gaster, 'Packing curves on surfaces with few intersections', Int. Math. Res. Not. IMRN (2017), rnx270.

[AL13] J. Aramayona and C. J. Leininger, 'Finite rigid sets in curve complexes', J. Topol. Anal. 5(2) (2013), 183-203.

[Bea83] A. F. Beardon, The Geometry of Discrete Groups, Graduate Texts in Mathematics, 91 (Springer, New York, 1983). 
[BBF15] M. Bestvina, K. Bromberg and K. Fujiwara, 'Constructing group actions on quasitrees and applications to mapping class groups', Publ. Math. Inst. Hautes Études Sci. 122(1) (2015), 1-64.

[BBM15] J. Birman, N. Broaddus and W. Menasco, 'Finite rigid sets and homologically nontrivial spheres in the curve complex of a surface', J. Topol. Anal. 7(1) (2015), 47-71.

[BM15] J. Birman and W. Menasco, 'The curve complex has dead ends', Geom. Dedicata 177 (2015), 71-74.

[Bow08] B. H. Bowditch, 'Tight geodesics in the curve complex', Invent. Math. 171(2) (2008), 281-300.

[BCM12] J. Brock, R. Canary and Y. Minsky, 'The classification of Kleinian surface groups, II: the ending lamination conjecture', Ann. of Math. (2) 176(1) (2012), 1-149.

[Bus92] P. Buser, Geometry and Spectra of Compact Riemann Surfaces, Progress in Mathematics, 106 (Birkhäuser Boston, Inc., Boston, MA, 1992).

[BP07] P. Buser and H. Parlier, 'The distribution of simple closed geodesics on a Riemann surface', in Complex Analysis and its Applications, OCAMI Stud., 2 (Osaka Munic. Univ. Press, Osaka, 2007), 3-10.

[CvL91] P. J. Cameron and J. H. van Lint, Designs, Graphs, Codes and their Links (Cambridge University Press, Cambridge, 1991).

[Chi72a] D. R. J. Chillingworth, 'Winding numbers on surfaces, I', Math. Ann. 196(3) (1972), 218-249.

[Chi72b] D. R. J. Chillingworth, 'Winding numbers on surfaces. II', Math. Ann. 199(3) (1972), 131-153.

[FMFPH ${ }^{+}$09] R. Fabila-Monroy, D. Flores-Peñaloza, C. Huemer, F. Hurtado, J. Urrutia and D. R. Wood, 'On the chromatic number of some flip graphs', Discrete Math. Theor. Comput. Sci. 11(2) (2009), 47-56.

[FM11] B. Farb and D. Margalit, A Primer on Mapping Class Groups (Princeton University Press, Princeton, NJ, 2011).

[GR13] C. Godsil and G. F. Royle, Algebraic Graph Theory, vol. 207 (Springer, New York, 2013).

[HS89] A. Haas and P. Susskind, 'The geometry of the hyperelliptic involution in genus two', Proc. Amer. Math. Soc. 105(1) (1989), 159-165.

[Har81] W. J. Harvey, 'Boundary structure of the modular group', in Riemann Surfaces and Related Topics: Proceedings of the 1978 Stony Brook Conference (State Univ. New York, Stony Brook, NY, 1978), Annals of Mathematics Studies, 97 (Princeton University Press, Princeton, NJ, 1981), 245-251.

[Irm15] I. Irmer, 'The Chillingworth class is a signed stable length', Algebr. Geom. Topol. 15(4) (2015), 1863-1876.

[Iva97] N. V. Ivanov, 'Automorphisms of complexes of curves and of Teichmüller spaces', in Progress in Knot Theory and Related Topics, Travaux en Cours, 56 (Hermann, Paris, 1997), 113-120.

[Joh80] D. Johnson, 'An abelian quotient of the mapping class group $\mathcal{I}_{g}$ ', Math. Ann. 249(3) (1980), 225-242.

[Joh83] D. Johnson, 'The structure of the Torelli group I: a finite set of generators for $\mathcal{J}$ ', Ann. of Math. (2) 118(3) (1983), 423-442.

[Joh85] D. Johnson, 'The structure of the Torelli group II: a characterization of the group generated by twists on bounding curves', Topology 24(2) (1985), 113-126. 
[JMM96] M. Juvan, A. Malnič and B. Mohar, 'Systems of curves on surfaces', J. Combin. Theory Ser. B 68(1) (1996), 7-22.

[KP02] R. P. Kent IV and D. Peifer, 'A geometric and algebraic description of annular braid groups', Internat. J. Algebra Comput. 12 (2002), 85-97.

[KK13] S.-H. Kim and T. Koberda, 'Right-angled Artin groups and finite subgraphs of curve graphs', Osaka J. Math. 53(3) (2016), 705-716.

[Kne55] M. Kneser, 'Aufgabe 360', Jahresber. Dtsch. Math.-Ver. 2 (1955), 27.

[Lee89] C. W. Lee, 'The associahedron and triangulations of the $n$-gon', European $J$. Combin. 10(6) (1989), 551-560.

[vLW01] J. H. van Lint and R. M. Wilson, A Course in Combinatorics (Cambridge University Press, Cambridge, 2001).

[Lov78] L. Lovász, 'Kneser's conjecture, chromatic number, and homotopy', J. Combin. Theory Ser. A 25(3) (1978), 319-324.

[MRT14] J. Malestein, I. Rivin and L. Theran, 'Topological designs', Geom. Dedicata 168 (2014), 221-233.

[MM99] H. A. Masur and Y. N. Minsky, 'Geometry of the complex of curves. I. Hyperbolicity', Invent. Math. 138(1) (1999), 103-149.

[Mat03] J. Matoušek, 'Using the Borsuk-Ulam Theorem', in Universitext, Lectures on topological methods in combinatorics and geometry, Written in cooperation with Anders Björner and Günter M. Ziegler (Springer-Verlag, Berlin, 2003), xii+196.

[MP78] W. H. Meeks III and J. Patrusky, 'Representing homology classes by embedded circles on a compact surface', Illinois J. Math. 22(2) (1978), 262-269.

[Min10] Y. Minsky, 'The classification of Kleinian surface groups. I. Models and bounds', Ann. of Math. (2) 171(1) (2010), 1-107.

[Prz15] P. Przytycki, 'Arcs intersecting at most once', Geom. Funct. Anal. 25(2) (2015), 658-670.

[Put08] A. Putman, 'A note on the connectivity of certain complexes associated to surfaces', Enseign. Math. (2) 54(34) (2008), 287-301.

[Put18] A. Putman, 'The Johnson homomorphism and its kernel', J. Reine Angew. Math. 735 (2018), 109-141.

[Sar11] S. Sarkar, 'Maslov index formulas for Whitney n-gons', J. Symplectic Geom. 9(2) (2011), 251-270.

[STT88] D. D. Sleator, R. E. Tarjan and W. P. Thurston, 'Rotation distance, triangulations, and hyperbolic geometry', J. Amer. Math. Soc. 1(3) (1988), 647-681. 